\title{
Modularity Results for Interpolation, Amalgamation and Superamalgamation
}

\author{
Silvio Ghilardi ${ }^{\mathrm{a}}$, Alessandro Gianola ${ }^{\mathrm{b}}$ \\ ${ }^{a}$ Università degli Studi di Milano, Dipartimento di Matematica, Milan, Italy \\ silvio.ghilardi@unimi.it \\ ${ }^{b}$ Free University of Bozen-Bolzano, Faculty of Computer Science, Bozen, Italy \\ gianola@inf.unibz.it
}

\begin{abstract}
Wolter in [38] proved that the Craig interpolation property transfers to fusion of normal modal logics. It is well-known [21] that for such logics Craig interpolation corresponds to an algebraic property called superamalgamability. In this paper, we develop model-theoretic techniques at the level of first-order theories in order to obtain general combination results transferring quantifier-free interpolation to unions of theories over non-disjoint signatures. Such results, once applied to equational theories sharing a common Boolean reduct, can be used to prove that superamalgamability is modular also in the non-normal case. We also state that, in such non-normal context, superamalgamability corresponds to a strong form of interpolation that we call "comprehensive interpolation property" (which consequently transfers to fusions).
\end{abstract}

Keywords: Interpolation, Fusion, Modal Logic, Superamalgamability

\section{Introduction}

Craig's interpolation theorem [7] is a model-theoretic result which applies to first order formulae and states that whenever a formula $\phi$ entails a formula $\psi$, then it is possible to find a third formula $\theta$ which can be interpolated beetwen $\phi$ and $\psi$, and which is defined over their common symbols. Interpolation theory has been recently introduced in verification, after the work of McMillan (see, e.g., [23]), and it has also a long tradition in non-classical logics (see for instance the seminal papers by L.L. Maksimova [20],[21]). In 
particular, the specific form of interpolation for modal logic is the following: a modal logic $L$ is said to enjoy the (local) interpolation property iff, whenever we consider two modal formulae $t_{1}$ and $t_{2}$ such that $\vdash_{L} t_{1} \rightarrow t_{2}$ holds, it is possible to find a modal formula $u$ such that (i) $\vdash_{L} t_{1} \rightarrow u$, (ii) $\vdash_{L} u \rightarrow t_{2}$, and (iii) the variables of $u$ are in common with both $t_{1}$ and $t_{2}$. In this context, of great importance is the study of combination of logics, focusing on the transfer of significant properties like interpolation. The simplest way of combining modal logics is given by the well-known notion of fusion: considering two modal logics $L_{1}$ and $L_{2}$ over the modal signatures $\Sigma_{M}^{1}$ and $\Sigma_{M}^{2}$ such that $\Sigma_{M}^{1} \cap \Sigma_{M}^{2}=\emptyset$, the fusion $L_{1} \oplus L_{2}$ is the least modal logic, over the modal signature $\Sigma_{M}^{1} \cup \Sigma_{M}^{2}$, that contains $L_{1} \cup L_{2}$.

In [38] Wolter proved that the fusion of two interpolating normal modal logics is also interpolating. However, the non-normal case remained open and in this paper we try to attack it: we show that superamalgamability transfers to fusions in the general non-normal context. It is well-known that superamalgamability (which is an algebraic condition) is equivalent to interpolation in the normal case [21]. Thus, our result implies Wolter's result and we prove that in the general non-normal case our result gives a fusion transfer theorem for a new strong form of interpolation (covering both local and global interpolation) which we call "comprehensive interpolation property".

The above result is obtained as a corollary of combination techniques for first-order theories: in fact, we specialize to the modal context modular conditions of combinability that generalize various previous works. The study of the modularity property of quantifier-free interpolation in first-order theories was first started in [40], where the disjoint signatures convex case was solved; in [5] - the journal version of [4] - the non-convex (still disjoint) case was also thoroughly investigated. In attacking combination problems for non-disjoint signatures, we follow the model-theoretic approach successfully employed in [11], [2], [14], [29], [26], [27], [28] for combined satisfiability; this approach relies on model-theoretic notions like $T_{0}$-compatibility.

The paper is organized in five sections. In Section 2, we introduce notations and basic ingredients concerning first order logic. In Section 3 we obtain a first general result (Theorem 3.1) which gives sufficient conditions for the transfer of quantifier-free interpolation in the non-disjoint signatures case; Theorem 3.1 has all known results for disjoint signatures case [5], [40] as an immediate consequence. In Section 4 we focus our attention on universal Horn theories, in order to obtain a modular condition (Theorem 4.1) referring to "minimal" amalgams. In Section 5, we apply Theorem 4.1 to modal logic: 
we prove that superamalgamability is a modular condition (Corollary 5.2), since it is equivalent to the combination condition of Theorem 4.1 in case the background theory is the theory of Boolean Algebras. Then, we syntactically characterize superamalgamability, by defining the notion of "comprehensive interpolation". Comprehensive interpolation, in the normal case, is nothing but standard interpolation, but in the non-normal case it looks like a stronger property, which transfers to fusions as a consequence of our results (Theorem 5.1).

\section{Formal Preliminaries in First Order Logic}

We adopt the usual first-order syntactic notions of signature, term, atom, (ground) formula, sentence, and so on. Let $\Sigma$ be a first-order signature; we assume the binary equality predicate symbol ' $=$ ' to be added to any signature (so, if $\Sigma=\emptyset$, then $\Sigma$ just contains equality). The signature obtained from $\Sigma$ by adding to it a set $\underline{a}$ of new constants (i.e., 0-ary function symbols) is denoted by $\Sigma \underline{a}$. A literal is an atomic formula or the negation of an atomic formula; a clause is a disjunction of literals and a positive clause is a disjunction of atoms. A formula is quantifier-free (or open) iff it does not contain quantifiers. A $\Sigma$-theory $T$ is a set of sentences (called the axioms of $T$ ) in the signature $\Sigma$ and it is universal iff it has universal closures of open formulae as axioms.

We also assume the usual first-order notion of interpretation and truth of a formula, with the proviso that the equality predicate $=$ is always interpreted as the identity relation. A formula $\varphi$ is satisfiable in $\mathcal{M}$ iff its existential closure is true in $\mathcal{M}$. A $\Sigma$-structure $\mathcal{M}$ is a model of a $\Sigma$-theory $T$ (in symbols $\mathcal{M} \models T$ ) iff all the sentences of $T$ are true in $\mathcal{M}$. If $\varphi$ is a formula, $T \models \varphi$ (' $\varphi$ is a logical consequence of $T$ ') means that the universal closure of $\varphi$ is true in all the models of $T$; $T$ is consistent iff it has a model. A sentence $\varphi$ is $T$-consistent iff $T \cup\{\varphi\}$ is consistent. A $\Sigma$-theory $T$ is complete iff for every $\Sigma$-sentence $\varphi$, either $\varphi$ or $\neg \varphi$ is a logical consequence of $T$. T admits quantifier elimination iff for every formula $\varphi(\underline{x})$ there is a quantifierfree formula $\varphi^{\prime}(\underline{x})$ such that $T \models \varphi(\underline{x}) \leftrightarrow \varphi^{\prime}(\underline{x})$ (notations like $\varphi(\underline{x})$ mean that $\varphi$ has free variables only among the tuple $\underline{x})$.

If $\Sigma_{0} \subseteq \Sigma$ is a subsignature of $\Sigma$ and if $\mathcal{M}$ is a $\Sigma$-structure, the $\Sigma_{0}$-reduct of $\mathcal{M}$ is the $\Sigma_{0}$-structure $\mathcal{M}_{\mid \Sigma_{0}}$ obtained from $\mathcal{M}$ by forgetting the interpretation of function and predicate symbols from $\Sigma \backslash \Sigma_{0}$. A $\Sigma$-homomorphism (or, simply, a homomorphism) between two $\Sigma$-structures $\mathcal{M}$ and $\mathcal{N}$ is any 
mapping $\mu:|\mathcal{M}| \longrightarrow|\mathcal{N}|$ among the support sets $|\mathcal{M}|$ of $\mathcal{M}$ and $|\mathcal{N}|$ of $\mathcal{N}$ satisfying the condition

$$
\mathcal{M}=\varphi \quad \Rightarrow \quad \mathcal{N} \models \varphi
$$

for all $\Sigma^{|\mathcal{M}|}$-atoms $\varphi$ (here $\mathcal{M}$ is regarded as a $\Sigma^{|\mathcal{M}|}$-structure, by interpreting each additional constant $a \in|\mathcal{M}|$ into itself and $\mathcal{N}$ is regarded as a $\Sigma^{|\mathcal{M}|_{-}}$ structure by interpreting each additional constant $a \in|\mathcal{M}|$ into $\mu(a)$ ). In case condition (1) holds for all $\Sigma^{|\mathcal{M}|}$-literals, the homomorphism $\mu$ is said to be an embedding and if it holds for all first order formulae, the embedding $\mu$ is said to be elementary. If $\mu: \mathcal{M} \longrightarrow \mathcal{N}$ is an embedding which is just the identity inclusion $|\mathcal{M}| \subseteq|\mathcal{N}|$, we say that $\mathcal{M}$ is a substructure of $\mathcal{N}$ or that $\mathcal{N}$ is an extension of $\mathcal{M}$. A $\Sigma$-structure $\mathcal{M}$ is said to be generated by a set $X$ included in its support $|\mathcal{M}|$ iff there are no proper substructures of $\mathcal{M}$ including $X$.

Given a signature $\Sigma$ and a $\Sigma$-structure $\mathcal{A}$, we indicate with $\Delta_{\Sigma}(\mathcal{A})$ the diagram of $\mathcal{A}$ : this is the set of sentences obtained by first expanding $\Sigma$ with a fresh constant $\bar{a}$ for every element $a$ from $|\mathcal{A}|$ and then taking the set of ground $\Sigma^{|\mathcal{A}|}$-literals which are true in $\mathcal{A}$ (under the natural expanded interpretation mapping $\bar{a}$ to $a$ ).

\subsection{Model completion and $T_{0}$-compatibility}

We recall a standard notion in Model Theory, namely the notion of a model completion of a first order theory [6] (we limit the definition to universal theories, because we shall use only this case):

Definition 2.1. Let $T_{0}$ be a universal $\Sigma_{0}$-theory and let $T_{0}^{\star} \supseteq T_{0}$ be a further $\Sigma_{0}$-theory; we say that $T_{0}^{\star}$ is a model completion of $T_{0}$ iff: (i) every model of $T_{0}$ can be embedded into a model of $T_{0}^{\star}$; (ii) for every model $\mathcal{M}$ of $T_{0}$, we have that $T_{0}^{\star} \cup \Delta_{\Sigma_{0}}(\mathcal{M})$ is a complete theory in the signature $\Sigma_{0}^{|\mathcal{M}|}$.

Since $T_{0}$ is universal, condition (ii) is equivalent to the fact that $T_{0}^{\star}$ has quantifier elimination; we recall also that the model completion $T_{0}^{\star}$ of a theory $T_{0}$ is unique, if it exists (see [6] for these results and for examples).

We also recall the concept of $T_{0}$-compatibility $[11,14]$, which is crucial for our combination technique.

Definition 2.2. Let $T$ be a theory in the signature $\Sigma$ and let $T_{0}$ be a universal theory in a subsignature $\Sigma_{0} \subseteq \Sigma$. We say that $T$ is $T_{0}$-compatible iff $T_{0} \subseteq T$ and there is a $\Sigma_{0}$-theory $T_{0}^{\star}$ such that: 
(i) $T_{0}^{\star}$ is a model completion of $T_{0}$;

(ii) every model of $T$ can be embedded, as a $\Sigma$-structure, into a model of $T \cup T_{0}^{\star}$.

Notice that if $T_{0}$ is the empty theory over the empty signature, then $T_{0}^{\star}$ is the theory axiomatizing an infinite domain, and the requirement of $T_{0^{-}}$ compatibility is equivalent to the stably infinite requirement of the NelsonOppen schema $[25,36]$ (in the sense that $T$ is $T_{0}$-compatible iff it is stably infinite). We remind that a theory $T$ is stably infinite iff every $T$-satisfiable quantifier-free formula (from the signature of $T$ ) is satisfiable in an infinite model of $T$. By compactness, it is possible to show that $T$ is stably infinite iff every model of $T$ embeds into an infinite one.

We underline that $T_{0}$-compatibility is a modular condition. The following result is proved in [11] (as Proposition 4.4):

Proposition 2.1. Let $T_{1}$ be a $\Sigma_{1}$-theory and let $T_{2}$ be $a \Sigma_{2}$-theory; suppose they are both compatible with respect to a $\Sigma_{0}$-theory $T_{0}$ (where $\Sigma_{0}:=\Sigma_{1} \cap \Sigma_{2}$ ). Then $T_{1} \cup T_{2}$ is $T_{0}$-compatible too.

\subsection{Interpolation and Amalgamation}

We say that a theory $T$ has quantifier-free interpolation iff the following hold, for every pair of quantifier free formulae $\varphi(\underline{x}, \underline{y}), \psi(y, \underline{z})$ : if $T \models$ $\varphi(\underline{x}, \underline{y}) \rightarrow \psi(\underline{y}, \underline{z})$, then there exists a quantifier-free formula $\theta(\underline{y})$ such that $T \models \varphi(\underline{x}, \underline{y}) \stackrel{\rightarrow}{\rightarrow}(\underline{y})$ and $T \models \theta(\underline{y}) \rightarrow \psi(\underline{y}, \underline{z})$. We underline that the requirement that $\theta$ is quantifier-free is essential: in general such a $\theta(y)$ exists by the Craig interpolation theorem, but it is not quantifier-free even if $\varphi, \psi$ are such.

Quantifier-free interpolation property can be semantically characterized using the following notions, introduced in $[3,5]$ (see [18] for several examples):

Definition 2.3. A theory $T$ has the sub-amalgamation property iff, for given models $\mathcal{M}_{1}$ and $\mathcal{M}_{2}$ of $T$ sharing a common substructure $\mathcal{A}$, there exists a further model $\mathcal{M}$ of $T$ endowed with embeddings $\mu_{1}: \mathcal{M}_{1} \longrightarrow \mathcal{M}$ and $\mu_{2}: \mathcal{M}_{2} \longrightarrow \mathcal{M}$ whose restrictions to the support of $\mathcal{A}$ coincide. The triple $\left(\mathcal{M}, \mu_{1}, \mu_{2}\right)$ (or, by abuse, $\mathcal{M}$ itself) is said to be a $T$-sub-amalgam of $\mathcal{M}_{1}, \mathcal{M}_{2}, \mathcal{A}$.

Definition 2.4. A theory $T$ has the strong sub-amalgamation property if the $T$-sub-amalgam $\left(\mathcal{M}, \mu_{1}, \mu_{2}\right)$ of $\mathcal{M}_{1}, \mathcal{M}_{2}, \mathcal{A}$ can be chosen so as to satisfy the 
following additional condition: if for some $m_{1}, m_{2}$ we have $\mu_{1}\left(m_{1}\right)=\mu_{2}\left(m_{2}\right)$, then there exists an element $a$ in $|\mathcal{A}|$ such that $m_{1}=a=m_{2}$.

If $T$ is universal, then every substructure of a model of $T$ is itself a model of $T$ : in these cases, we shall drop the prefix sub- and directly speak of 'amalgamability', 'strong amalgamability' and ' $T$-amalgam'. The following fact is proved in [5], as Theorem 3.3:

Theorem 2.1. A theory $T$ has the sub-amalgamation property iff it admits quantifier-free interpolants.

\section{Conditions for Combination}

The main result from [5] says that if $T_{1}, T_{2}$ have disjoint signatures, are both stably infinite and both enjoy the strong sub-amalgamation property, then the combined theory $T_{1} \cup T_{2}$ also has the strong sub-amalgamation property $^{1}$ (and so it has quantifier-free interpolation).

In this paper, we try to extend the above results to the non-disjoint signatures case. The idea, already shown to be fruitful for combined satisfiability problems in [11], is to use $T_{0}$-compatibility as the proper generalization of stable infiniteness.

We shall first obtain a rather abstract sufficient condition for the transfer of quantifier-free interpolation property to combined theories; nevertheless, we show that such a sufficient condition generalizes the disjoint signatures result from [5]. Then we move to the case in which the shared theory $T_{0}$ is Horn and obtain as a corollary a specialized result which is quite effective in modal logic applications.

\subsection{Sub-amalgamation schemata}

Let $T_{0}, T$ be theories in their respective signatures $\Sigma_{0}, \Sigma$ such that $\Sigma_{0} \subseteq \Sigma$, $T_{0}$ is universal and $T_{0} \subseteq T$. If $\mathcal{M}_{1}$ and $\mathcal{M}_{2}$ are $\Sigma$-models of $T$ with a common substructure $\mathcal{A}$, we call the triple $\left(\mathcal{M}_{1}, \mathcal{M}_{2}, \mathcal{A}\right)$ a $T$-fork (or, simply, a fork).

\footnotetext{
${ }^{1}$ It is possible to characterize syntactically strong sub-amalgamability in terms of a suitable 'equality interpolating' condition [5]. That sub-amalgamability needs to be strenghtened to strong sub-amalgamability in order to get positive combination results is demonstrated by converse facts also proved in [5].
} 
The sub-amalgamation schema $\sigma_{T_{0}}^{T}$ (of $T$ over $T_{0}$ ) is the following function, associating sets of $T_{0}$-amalgams with $T$-forks: ${ }^{2}$

$\sigma_{T_{0}}^{T}\left[\left(\mathcal{M}_{1}, \mathcal{M}_{2}, \mathcal{A}\right)\right]:=\left\{\begin{array}{c}\text { the set of all }\left(\mathcal{B}, \nu_{1}, \nu_{2}\right) \text { s.t. } \\ \text { (i) }\left(\mathcal{B}, \nu_{1}, \nu_{2}\right) \text { is a } T_{0} \text {-amalgam of the } \Sigma_{0} \text {-reducts of } \\ \mathcal{M}_{1} \text { and } \mathcal{M}_{2} \text { over the } \Sigma_{0} \text {-reduct of } \mathcal{A} \\ \text { (ii) } \mathcal{B} \text { is generated, as a } \Sigma_{0} \text {-structure, by the union } \\ \text { of the images of } \nu_{1} \text { and } \nu_{2} ; \\ \text { (iii) }\left(\mathcal{B}, \nu_{1}, \nu_{2}\right) \text { is embeddable in the } \Sigma_{0} \text {-reduct of a } \\ T \text {-sub-amalgam of the fork }\left(\mathcal{M}_{1}, \mathcal{M}_{2}, \mathcal{A}\right) .\end{array}\right\}$

Condition (iii) means that there is a $T$-sub-amalgam $\left(\mathcal{M}, \mu_{1}, \mu_{2}\right)$ such that $\mathcal{B}$ is a $\Sigma_{0}$-substructure of $\mathcal{M}$ and that $\mu_{1}, \mu_{2}$ coincide with $\nu_{1}, \nu_{2}$ on their domains.

Condition (ii) ensures that, disregarding isomorphic copies, $\sigma_{T_{0}}^{T}\left[\left(\mathcal{M}_{1}, \mathcal{M}_{2}, \mathcal{A}\right)\right]$ is a set and not a proper class. Recall that $T_{0}$ is universal, so that substructures of models of $T_{0}$ are also models of $T_{0}$. This ensures that the following Proposition trivially holds:

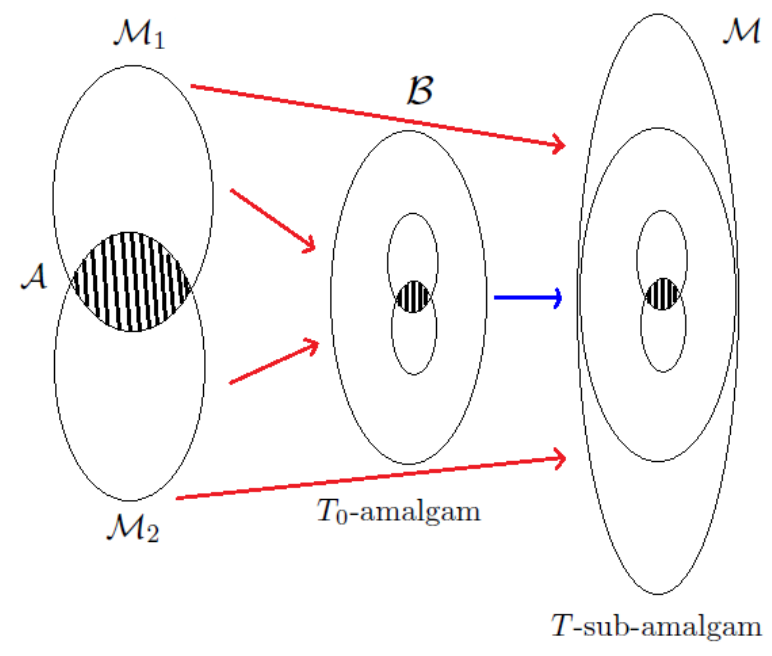

\footnotetext{
${ }^{2}$ It is not difficult to realize (using well-known Löwenheim-Skolem theorems [6]) that one can get all the results in the paper by limiting this definition to forks among structures whose cardinality is bounded by the cardinality of the set of the formulae in our signatures (signatures are finite or countable in all practical cases).
} 
Proposition 3.1. $T$ is sub-amalgamable iff $\sigma_{T_{0}}^{T}$ is not empty (i.e. iff we have that $\sigma_{T_{0}}^{T}\left[\left(\mathcal{M}_{1}, \mathcal{M}_{2}, \mathcal{A}\right)\right] \neq \emptyset$, for all forks $\left.\left(\mathcal{M}_{1}, \mathcal{M}_{2}, \mathcal{A}\right)\right)$.

We are ready to formulate the main combination result, which is the following Theorem: one side of the inclusion of the statement is immediate, whereas, for the other one, $T_{0}$-compatibility is needed.

Theorem 3.1 (Combination Theorem). Let $T_{1}$ and $T_{2}$ be two theories in their respective signatures $\Sigma_{1}, \Sigma_{2}$; assume that they are both $T_{0}$-compatible where $T_{0}$ is a further universal theory in the signature $\Sigma_{0}:=\Sigma_{1} \cap \Sigma_{2}$. The following holds for the sub-amalgamation schema of $T_{1} \cup T_{2}$ over $T_{0}$ :

$$
\sigma_{T_{0}}^{T_{1} \cup T_{2}}\left[\left(\mathcal{M}_{1}, \mathcal{M}_{2}, \mathcal{A}\right)\right]=\sigma_{T_{0}}^{T_{1}}\left[\left(\mathcal{M}_{1}, \mathcal{M}_{2}, \mathcal{A}\right)_{\mid \Sigma_{1}}\right] \cap \sigma_{T_{0}}^{T_{2}}\left[\left(\mathcal{M}_{1}, \mathcal{M}_{2}, \mathcal{A}\right)_{\mid \Sigma_{2}}\right]
$$

for every $\left(T_{1} \cup T_{2}\right)$-fork $\left(\mathcal{M}_{1}, \mathcal{M}_{2}, \mathcal{A}\right)$ (here, with $\left(\mathcal{M}_{1}, \mathcal{M}_{2}, \mathcal{A}\right)_{\mid \Sigma_{i}}$ we indicate the $T_{i}$-fork obtained by taking reducts to the signature $\left.\Sigma_{i}\right)$.

Proof of Combination Theorem. The proof of Theorem 3.1 splits into a few steps. We begin by recalling some standard results from model theory and by introducing some preliminary lemmata. The following easy fact is proved in [5], as Lemma 3.7:

Lemma 3.1. Let $\Sigma_{1}, \Sigma_{2}$ be two signatures and $\mathcal{A}$ be a $\Sigma_{1} \cup \Sigma_{2}$-structure; then $\Delta_{\Sigma_{1} \cup \Sigma_{2}}(\mathcal{A})$ is logically equivalent to $\Delta_{\Sigma_{1}}(\mathcal{A}) \cup \Delta_{\Sigma_{2}}(\mathcal{A})$.

Proof. As a general fact, let us first observe that every diagram is equivalent to its flat subdiagram, in the following sense. Let $\mathcal{B}$ be a $\Sigma$-structure; the flat $\Sigma$-subdiagram of $\mathcal{B}$ is the set $\Delta_{\Sigma}^{f}(\mathcal{B})$ of literals of the kind

$$
f\left(a_{1}, \ldots, a_{n}\right)=b, \quad a_{1} \neq a_{2}, \quad P\left(a_{1}, \ldots, a_{n}\right), \quad \neg P\left(a_{1}, \ldots, a_{n}\right)
$$

that are true in $\mathcal{B}$ (here $a_{1}, \ldots, a_{n}, b$ are free constants naming elements from $|\mathcal{B}|)$. That every diagram is equivalent to its flat subdiagram can be easily proved from the fact (to be shown by induction) that for every $\Sigma \cup|\mathcal{B}|$-ground term $t$ there is $a \in|\mathcal{B}|$ such that $\Delta_{\Sigma}^{f}(\mathcal{B}) \vdash t=a$.

Now we have that $\Delta_{\Sigma_{1} \cup \Sigma_{2}}(\mathcal{A})$ is logically equivalent to $\Delta_{\Sigma_{1} \cup \Sigma_{2}}^{f}(\mathcal{A})$ and the latter is $\Delta_{\Sigma_{1}}^{f}(\mathcal{A}) \cup \Delta_{\Sigma_{2}}^{f}(\mathcal{A})$ which in turn is equivalent to $\Delta_{\Sigma_{1}}(\mathcal{A}) \cup \Delta_{\Sigma_{2}}(\mathcal{A})$.

An easy but nevertheless important basic result, called the Robinson Diagram Lemma [6], says that, given any $\Sigma$-structure $\mathcal{B}$, the embeddings 
$\mu: \mathcal{A} \longrightarrow \mathcal{B}$ are in bijective correspondence with expansions of $\mathcal{B}$ to $\Sigma^{|\mathcal{A}|_{-}}$ structures which are models of $\Delta_{\Sigma}(\mathcal{A})$. The expansions and the embeddings are related in the obvious way: $\bar{a}$ is interpreted as $\mu(a)$.

The following Lemma is proved using this property of diagrams:

Lemma 3.2. Let $T_{0}, T$ be theories in their respective signatures $\Sigma_{0}, \Sigma$ such that $\Sigma_{0} \subseteq \Sigma$ and $T_{0} \subseteq T$; let $\left(\mathcal{M}_{1}, \mathcal{M}_{2}, \mathcal{A}\right)$ be a T-fork. For a $T_{0}$-amalgam $\left(\mathcal{B}, \nu_{1}, \nu_{2}\right)$ the following conditions are equivalent (we suppose that the support of $\mathcal{B}$ is disjoint from the supports of $\left.\mathcal{M}_{1}, \mathcal{M}_{2}\right)$ :

(i) $\left(\mathcal{B}, \nu_{1}, \nu_{2}\right) \in \sigma_{T_{0}}^{T}\left[\left(\mathcal{M}_{1}, \mathcal{M}_{2}, \mathcal{A}\right)\right]$;

(ii) the following theory $(*)$ is consistent

$$
\begin{aligned}
& T \cup \Delta_{\Sigma}\left(\mathcal{M}_{1}\right) \cup \Delta_{\Sigma}\left(\mathcal{M}_{2}\right) \cup \Delta_{\Sigma_{0}}(\mathcal{B}) \cup \\
& \cup\left\{\bar{a}_{1}=\bar{b} \| b \in|\mathcal{B}|, a_{1} \in\left|\mathcal{M}_{1}\right|, \nu_{1}\left(a_{1}\right)=b\right\} \cup \\
& \cup\left\{\bar{a}_{2}=\bar{b} \| b \in|\mathcal{B}|, a_{2} \in\left|\mathcal{M}_{2}\right|, \nu_{2}\left(a_{2}\right)=b\right\}
\end{aligned}
$$

Furthermore, in case $T$ is $T_{0}$-compatible, we can equivalently replace $T$ by $T \cup T_{0}^{\star}$ in the theory $(*)$ mentioned in (ii) above.

Proof. By the above mentioned property of diagrams, the consistency of $(*)$ means that there is a model $\mathcal{N} \models T$ and there are three embeddings

$$
\mu_{1}: \mathcal{M}_{1} \longrightarrow \mathcal{N}, \quad \mu_{2}: \mathcal{M}_{2} \longrightarrow \mathcal{N}, \quad \nu: \mathcal{B} \longrightarrow \mathcal{N}
$$

(the last one is a $\Sigma_{0}$-embedding, the first two are $\Sigma$-embeddings) such that $\nu \circ \nu_{1}=\mu_{1}$ and $\nu \circ \nu_{2}=\mu_{2}$. Since $\mu_{1}, \mu_{2}$ agree on the support of $\mathcal{A}$, the triple $\left(\mathcal{N}, \mu_{1}, \mu_{2}\right)$ is a $T$-sub-amalgam of the fork. To make $\mathcal{B}$ a substructure of $\mathcal{N}$, it is sufficient to make a renaming of the elements in the image of $\nu$ (so that $\nu$ becomes an inclusion). Thus consistency of $(*)$ means precisely that $\left(\mathcal{B}, \nu_{1}, \nu_{2}\right) \in \sigma_{T_{0}}^{T}\left[\left(\mathcal{M}_{1}, \mathcal{M}_{2}, \mathcal{A}\right)\right]$.

Since, by $T_{0}$-compatibility, every model of $T$ can be embedded into a model of $T \cup T_{0}^{\star}$, the consistency of $(*)$ is the same of the consistency of $T_{0}^{\star} \cup(*)$.

We need a further result from model theory to be found in textbooks like [6]; it can be seen as a combination result 'ante litteram': 
Lemma 3.3 (Joint Consistency). Let $\Theta_{1}, \Theta_{2}$ be two signatures and let $\Theta_{0}:=$ $\Theta_{1} \cap \Theta_{2}$; suppose that the $\Theta_{1}$-theory $U_{1}$ and the $\Theta_{2}$-theory $U_{2}$ are both consistent and that there is a $\Theta_{0}$-theory $U_{0}$ which is complete and included both in $U_{1}$ and in $U_{2}$. Then, $U_{1} \cup U_{2}$ is also consistent.

Proof. There are basically two proofs of this result, one by Craig's interpolation Theorem and another one by a double chain argument. The interested reader is referred to $[6]$.

We can now prove Theorem 3.1; the Theorem concerns theories $T_{1}, T_{2}$ (in their respective signatures $\Sigma_{1}, \Sigma_{2}$ ) which are both $T_{0}$-compatible with respect to a universal theory $T_{0}$ in the shared signature $\Sigma_{0}:=\Sigma_{1} \cap \Sigma_{2}$.

Fix a $T_{1} \cup T_{2}$-fork $\left(\mathcal{M}_{1}, \mathcal{M}_{2}, \mathcal{A}\right)$. On one side, it is evident that if $\left(\mathcal{B}, \nu_{1}, \nu_{2}\right)$ belongs to $\sigma_{T_{0}}^{T_{1} \cup T_{2}}\left[\left(\mathcal{M}_{1}, \mathcal{M}_{2}, \mathcal{A}\right)\right]$, then it also belongs to $\sigma_{T_{0}}^{T_{1}}\left[\left(\mathcal{M}_{1}, \mathcal{M}_{2}, \mathcal{A}\right)_{\mid \Sigma_{1}}\right] \cap$ $\sigma_{T_{0}}^{T_{2}}\left[\left(\mathcal{M}_{1}, \mathcal{M}_{2}, \mathcal{A}\right)_{\mid \Sigma_{2}}\right]$

Vice versa, suppose that $\left(\mathcal{B}, \nu_{1}, \nu_{2}\right)$ belongs to $\sigma_{T_{0}}^{T_{1}}\left[\left(\mathcal{M}_{1}, \mathcal{M}_{2}, \mathcal{A}\right)_{\mid \Sigma_{1}}\right]$ and to $\sigma_{T_{0}}^{T_{2}}\left[\left(\mathcal{M}_{1}, \mathcal{M}_{2}, \mathcal{A}\right)_{\mid \Sigma_{2}}\right] ;$ in order to show that it belongs to $\sigma_{T_{0}}^{T_{1} \cup T_{2}}\left[\left(\mathcal{M}_{1}, \mathcal{M}_{2}, \mathcal{A}\right)\right]$, in view of Lemmas 3.1 and 3.2 (recall also Proposition 2.1), we need to show that the following theory (let us call it $U$ ) is consistent:

$$
\begin{aligned}
& T_{1} \cup T_{2} \cup T_{0}^{\star} \cup \Delta_{\Sigma_{1}}\left(\mathcal{M}_{1}\right) \cup \Delta_{\Sigma_{1}}\left(\mathcal{M}_{2}\right) \cup \Delta_{\Sigma_{0}}(\mathcal{B}) \cup \\
& \cup \Delta_{\Sigma_{2}}\left(\mathcal{M}_{1}\right) \cup \Delta_{\Sigma_{2}}\left(\mathcal{M}_{2}\right) \cup \\
& \cup\left\{\bar{a}_{1}=\bar{b} \| b \in|\mathcal{B}|, a_{1} \in\left|\mathcal{M}_{1}\right|, \nu_{1}\left(a_{1}\right)=b\right\} \cup \\
& \cup\left\{\bar{a}_{2}=\bar{b} \| b \in|\mathcal{B}|, a_{2} \in\left|\mathcal{M}_{2}\right|, \nu_{2}\left(a_{2}\right)=b\right\} .
\end{aligned}
$$

The idea is to use Robinson Joint Consistency Lemma 3.3 and split $U$ as $U_{1} \cup U_{2}$. Now $U$ is a theory in the signature $\Sigma_{1} \cup \Sigma_{2} \cup\left|\mathcal{M}_{1}\right| \cup\left|\mathcal{M}_{2}\right| \cup|\mathcal{B}|$; we let (for $i=1,2$ ) $U_{i}$ be the following theory in the signature $\Sigma_{i} \cup\left|\mathcal{M}_{1}\right| \cup\left|\mathcal{M}_{2}\right| \cup|\mathcal{B}|$ :

$$
\begin{aligned}
& T_{i} \cup T_{0}^{\star} \cup \Delta_{\Sigma_{i}}\left(\mathcal{M}_{1}\right) \cup \Delta_{\Sigma_{i}}\left(\mathcal{M}_{2}\right) \cup \Delta_{\Sigma_{0}}(\mathcal{B}) \cup \\
& \cup\left\{\bar{a}_{1}=\bar{b} \| b \in|\mathcal{B}|, a_{1} \in\left|\mathcal{M}_{1}\right|, \nu_{1}\left(a_{1}\right)=b\right\} \cup \\
& \cup\left\{\bar{a}_{2}=\bar{b} \| b \in|\mathcal{B}|, a_{2} \in\left|\mathcal{M}_{2}\right|, \nu_{2}\left(a_{2}\right)=b\right\} .
\end{aligned}
$$

Notice that $U_{i}$ is consistent by Lemma 3.2 because our assumption is that $\left(\mathcal{B}, \nu_{1}, \nu_{2}\right)$ belongs to $\sigma_{T_{0}}^{T_{i}}\left[\left(\mathcal{M}_{1}, \mathcal{M}_{2}, \mathcal{A}\right)_{\mid \Sigma_{i}}\right]$. We now only have to identify a complete theory $U_{0}$ included in $U_{1} \cap U_{2}$. The shared signature of $U_{1}$ and $U_{2}$ 
is $\Sigma_{0} \cup\left|\mathcal{M}_{1}\right| \cup\left|\mathcal{M}_{2}\right| \cup|\mathcal{B}|$ and we take as $U_{0}$ the theory

$$
\begin{aligned}
& T_{0}^{\star} \cup \Delta_{\Sigma_{0}}\left(\mathcal{M}_{1}\right) \cup \Delta_{\Sigma_{0}}\left(\mathcal{M}_{2}\right) \cup \Delta_{\Sigma_{0}}(\mathcal{B}) \cup \\
& \cup\left\{\bar{a}_{1}=\bar{b} \| b \in|\mathcal{B}|, a_{1} \in\left|\mathcal{M}_{1}\right|, \nu_{1}\left(a_{1}\right)=b\right\} \cup \\
& \cup\left\{\bar{a}_{2}=\bar{b} \| b \in|\mathcal{B}|, a_{2} \in\left|\mathcal{M}_{2}\right|, \nu_{2}\left(a_{2}\right)=b\right\} .
\end{aligned}
$$

By the definition of a model-completion ( $T_{0}^{\star}$ is a model-completion of $T_{0}$ ), we know that $T_{0}^{\star} \cup \Delta_{\Sigma_{0}}(\mathcal{B})$ is a complete theory in the signature $\Sigma_{0} \cup|\mathcal{B}|$. Now it is sufficient to observe that every $\Sigma_{0} \cup\left|\mathcal{M}_{1}\right| \cup\left|\mathcal{M}_{2}\right| \cup|\mathcal{B}|$-sentence is equivalent, modulo $U_{0} \supseteq T_{0}^{\star} \cup \Delta_{\Sigma_{0}}(\mathcal{B})$, to a $\Sigma_{0} \cup|\mathcal{B}|$-sentence: this is clear because $U_{0}$ contains the sentences

$$
\begin{aligned}
& \left\{\bar{a}_{1}=\bar{b} \| b \in|\mathcal{B}|, a_{1} \in\left|\mathcal{M}_{1}\right|, \nu_{1}\left(a_{1}\right)=b\right\} \cup \\
& \cup\left\{\bar{a}_{2}=\bar{b} \| b \in|\mathcal{B}|, a_{2} \in\left|\mathcal{M}_{2}\right|, \nu_{2}\left(a_{2}\right)=b\right\} .
\end{aligned}
$$

which can be used to eliminate the constants from $\left|\mathcal{M}_{1}\right| \cup\left|\mathcal{M}_{2}\right|$.

Despite its abstract formulation, Theorem 3.1 is powerful enough to imply the main disjoint signatures result of [5] and also to work out interesting examples arising from software verification: in [12] it is shown how to use Theorem 3.1 to prove that suitable unions of theories describing data structures (like lists and binary trees) are quantifier-free interpolating. We explain in more detail how to derive the disjoint signatures result of [5] from Theorem 3.1:

Example 3.1 (The disjoint signatures case). Let $S_{0}, S_{1}, S_{2}$ be sets such that $S_{0} \subseteq S_{1}, S_{0} \subseteq S_{2}$; the amalgamated sum $S_{1}+_{S_{0}} S_{2}$ of $S_{1}, S_{2}$ over $S_{0}$ is just the set-theoretic union $S_{1} \cup S_{2}$ in which elements from $S_{1} \backslash S_{0}$ are renamed away so as to be different from the elements of $S_{2} \backslash S_{0}$. With this terminology, a theory $T$ is strongly sub-amalgamable iff its sub-amalgamation schema over the empty theory $T_{0}$ is such that $\sigma_{T_{0}}^{T}\left[\left(\mathcal{M}_{1}, \mathcal{M}_{2}, \mathcal{A}\right)\right]$ always contains the amalgamated sum of the supports of $\mathcal{M}_{1}, \mathcal{M}_{2}$ over the support of $\mathcal{A}$. Thus, Theorem 3.1 says in particular that if $T_{1}, T_{2}$ are both stably infinite and strongly sub-amalgamable, then so is $T_{1} \cup T_{2}$ (and the last theory is in particular quantifier-free interpolating).

\section{When the shared theory is Horn}

Theorem 3.1 gives modular information to determine the combined subamalgamation schema, but it is not a modular result itself. In fact, a modular 
result should identify a condition $C$ on a single (standing alone) theory such that whenever $T_{1}, T_{2}$ satisfy $C$, then $T_{1} \cup T_{2}$ also satisfies $C$. To get a modular sufficient condition for quantifier-free interpolation, we need to specialize our framework. In doing that, we are still guided by what happens in the disjoint signatures case. Although satisfactory results could be obtained without Horn hypotheses (see for a reference [15]), we prefer to assume here that the shared theory is universal Horn, on one side in order to simplify the statement of the results below, on the other side because universal Horn theories are sufficient for our applications to Modal Logic. Recall that a $\Sigma$-theory $T$ is universal Horn iff it can be axiomatized via Horn clauses (i.e. via formulae of the form $A_{1} \wedge \cdots \wedge A_{n} \rightarrow B$, where the $A_{i}$ are atoms and $B$ is either an atom or $\perp$ ). For simplicity (and because our applications to modal logic do not require more), in this section we shall consider only universal Horn theories axiomatized by clauses containing exactly one positive literal. We fix such a $T$ for the remaining part of this section (we let also $\Sigma$ be the signature of $T$ ).

\subsection{Minimal amalgam and presentations}

In universal Horn theories, it is possible to show that if amalgamation holds, then there is always a (unique up to isomorphisms) minimal amalgam this fact is basically due to the universal property of pushouts. We introduce the relevant notions:

Definition 4.1. Let $\left(\mathcal{M}_{1}, \mathcal{M}_{2}, \mathcal{A}\right)$ be a $T$-fork; a pushout of the fork is a triple $\left(\mathcal{M}, \mu_{1}, \mu_{2}\right)$, where $\mathcal{M}$ is a $T$-model and $\mu_{1}: \mathcal{M}_{1} \longrightarrow \mathcal{M}, \mu_{2}: \mathcal{M}_{2} \longrightarrow$ $\mathcal{M}$ are $\Sigma$-homomorphisms whose restrictions to the support of $\mathcal{A}$ coincide, such that for every other triple $\left(\mathcal{M}^{\prime}, \mu_{1}^{\prime}, \mu_{2}^{\prime}\right)$ with the same properties, there is a unique homomorphism (called the comparison homomorphism) $\theta: \mathcal{M} \longrightarrow$ $\mathcal{M}^{\prime}$ such that $\theta \circ \mu_{i}=\mu_{i}^{\prime}(i=1,2)$.

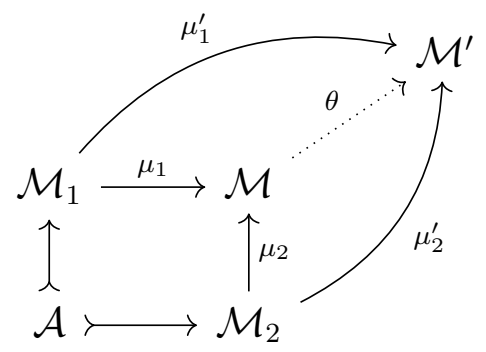

Definition 4.2. If the pushout $\left(\mathcal{M}, \mu_{1}, \mu_{2}\right)$ of the $T$-fork $\left(\mathcal{M}_{1}, \mathcal{M}_{2}, \mathcal{A}\right)$ is an amalgam (i.e. if $\mu_{1}, \mu_{2}$ are monomorphisms), it is called the minimal $T$-amalgam of the $T$-fork. 
Notice that, even when the pushout is an amalgam, comparison morphisms need not be injective.

Pushouts of $T$-forks (more generally, of $T$-spans) always exist: this is essentially a consequence of well-known co-completeness results for our categories of models [1], nevertheless we shall use an explicit construction in the sequel (it will be useful in our application to modal logic).

The key feature of $T$ is that it admits presentations, in the following sense. A $T$-presentation is a pair $(X, \Gamma)$, where $X$ is a set of fresh constants and $\Gamma$ is a set of $\Sigma^{X}$-atoms. To a $T$-presentation we associate the $T$-model $F_{T}(X, \Gamma)$ built as follows:

(i) the support $\left|F_{T}(X, \Gamma)\right|$ of $F_{T}(X, \Gamma)$ is formed by the equivalence classes of $\Sigma^{X}$-ground terms with respect to the equivalence relation $\sim_{\Gamma}$ defined by: $t \sim_{\Gamma} u$ iff $T \cup \Gamma \models t=u$;

(ii) function and relation symbols are interpreted so as to have that $F_{T}(X, \Gamma) \models$ $A$ holds iff $T \cup \Gamma \models A$, for every $\Sigma^{X}$-atom $A$ (clearly $F_{T}(X, \Gamma)$ is a $\Sigma^{X}$ structure, with constants from $X$ interpreted as their own equivalence classes)..$^{3}$

Thanks to the current assumption on $T$ (i.e. that the axioms of $T$ are all Horn clauses with exactly one positive literal), we can easily check that $F_{T}(X, \Gamma) \models$ $T$.

The fundamental property of $F_{T}(X, \Gamma) \models T$ is the following (this is very similar to the diagrams property): for every $T$-model $\mathcal{M}$, there is a bijective correspondence between $\Sigma$-homomorphisms $F_{T}(X, \Gamma) \longrightarrow \mathcal{M}$ and expansions of $\mathcal{M}$ to $\Sigma^{X}$-structures which are models of $\Gamma$.

Every model $\mathcal{M}$ of $T$ is isomorphic to a model of the kind $F_{T}(X, \Gamma)$ : this is because it is easily seen that $\mathcal{M} \simeq F_{T}\left(|\mathcal{M}|, \Delta_{\Sigma}^{+}(\mathcal{M})\right.$ ), where $\Delta_{\Sigma}^{+}(\mathcal{M})$ (the positive diagram of $\mathcal{M})$ is given by $\left\{A \mid A\right.$ is an atomic formula of $\Sigma^{|\mathcal{M}|}$ s.t. $\mathcal{M}=$ $A\}$. We call $F_{T}\left(|\mathcal{M}|, \Delta_{\Sigma}^{+}(\mathcal{M})\right)$ the canonical presentation of $\mathcal{M}$.

Existence of minimal amalgam for any universal Horn theory $T$ having the amalgamation property is an immediate consequence of the following:

\footnotetext{
${ }^{3}$ In more detail, in $F_{T}(X, \Gamma)$ an $n$-ary function symbol $f$ is interpreted as the function mapping the tuple of equivalence classes $\left[t_{1}\right], \ldots,\left[t_{n}\right]$ to the equivalence class $\left[f\left(t_{1}, \ldots, t_{n}\right)\right]$; an $n$-ary relation symbol $R$ is interpreted as the set of tuples of equivalence classes $\left[t_{1}\right], \ldots,\left[t_{n}\right]$ such that $T \cup \Gamma \models R\left(t_{1}, \ldots, t_{n}\right)$.
} 
Proposition 4.1. Every $T$-fork $\left(\mathcal{M}_{1}, \mathcal{M}_{2}, \mathcal{A}\right)$ has a pushout $\left(\mathcal{M}, \mu_{1}, \mu_{2}\right)$; if $T$ has the amalgamation property, the pushout is the minimal T-amalgam of the T-fork.

Proof. Up to renamings, we can freely suppose that $\left|\mathcal{M}_{1}\right| \cap\left|\mathcal{M}_{2}\right|=|\mathcal{A}|$. Let us take

$$
\mathcal{M}:=F_{T}\left(\left|\mathcal{M}_{1}\right| \cup\left|\mathcal{M}_{2}\right|, \Delta_{\Sigma}^{+}\left(\mathcal{M}_{1}\right) \cup \Delta_{\Sigma}^{+}\left(\mathcal{M}_{2}\right)\right)
$$

and let $\mu_{1}$ (resp. $\mu_{2}$ ) be the map associating an element from $\left|\mathcal{M}_{1}\right|$ (resp. $\left.\left|\mathcal{M}_{2}\right|\right)$ to its own equivalence class in $\mathcal{M}$. The fact that this is a pushout is guaranteed by the fundamental property of the presentations: indeed, given a $T$-model $\mathcal{M}^{\prime}$, the $\Sigma$-homomorphisms $\mathcal{M} \longrightarrow \mathcal{M}^{\prime}$ are in bijective correspondence with expansions of $\mathcal{M}^{\prime}$ to a $\Sigma^{\left|\mathcal{M}_{1}\right| \cup\left|\mathcal{M}_{2}\right|}$-structure modeling $\Delta_{\Sigma}^{+}\left(\mathcal{M}_{1}\right) \cup \Delta_{\Sigma}^{+}\left(\mathcal{M}_{2}\right)$ and the latter are in bijective correspondence with pairs of $\Sigma$-homomorphisms $\mathcal{M}_{1} \longrightarrow \mathcal{M}^{\prime}$ and $\mathcal{M}_{2} \longrightarrow \mathcal{M}^{\prime}$, agreeing on $\left|\mathcal{M}_{1}\right| \cap$ $\left|\mathcal{M}_{2}\right|=|\mathcal{A}|{ }^{4}$

If $T$ has the amalgamation property, then there exists a $T$-amalgam $\mathcal{N}$, with embeddings $\nu_{i}: \mathcal{M}_{i} \longrightarrow \mathcal{N}(i=1,2)$; by the above property of the pushout, there is $\theta: \mathcal{M} \longrightarrow \mathcal{N}$ with $\nu_{i}=\theta \circ \mu_{i}(i=1,2)$. Since the $\nu_{i}$ are injective and $\nu_{i}=\theta \circ \mu_{i}$, then also the $\mu_{i}$ are injective; the same argument shows that the $\mu_{i}$ reflect relations and hence they are embeddings: if a relation $R$ holds in $\mathcal{M}$ (when applied to some parameters from $\mathcal{M}_{i}$ ), then it holds in $\mathcal{N}$ because $\theta$ is a homomorphism, hence it holds also in $\mathcal{M}_{i}$, since the $\nu_{i}$ are embeddings. Thus, if $T$ has the amalgamation property, the pushout (2) is an amalgam (minimal by the definition of pushout) of the fork $\left(\mathcal{M}_{1}, \mathcal{M}_{2}, \mathcal{A}\right)$.

It is useful to have a formula like (2) operating in the case where we are given $T$-presentations of $\mathcal{M}_{1}, \mathcal{M}_{2}$ which might not be the canonical ones. We first introduce $T$-presentations of embeddings and of $T$-forks.

Suppose that we are given an embedding among $T$-models; up to an isomorphism, we can assume that it is a substructure inclusion $\mathcal{M}_{1} \subseteq \mathcal{M}_{2}$. Passing to canonical presentations, again up to isomorphisms, we have an embedding

$$
F_{T}\left(\left|\mathcal{M}_{1}\right|, \Delta_{\Sigma}^{+}\left(\mathcal{M}_{1}\right)\right) \longrightarrow F_{T}\left(\left|\mathcal{M}_{2}\right|, \Delta_{\Sigma}^{+}\left(\mathcal{M}_{2}\right)\right)
$$

\footnotetext{
${ }^{4}$ A slight modification of this construction shows the existence of pushouts also when the maps of $\mathcal{A}$ into $\mathcal{M}_{i}$ are $\Sigma$-homomorphisms (not just substructure inclusions).
} 
which is also 'canonical', in the sense that for every $a \in\left|\mathcal{M}_{1}\right|$, the embedding (3) maps the equivalence class of $a$ in $F\left(\left|\mathcal{M}_{1}\right|, \Delta_{\Sigma}^{+}\left(\mathcal{M}_{1}\right)\right)$ into the equivalence class of $a$ in $F\left(\left|\mathcal{M}_{2}\right|, \Delta_{\Sigma}^{+}\left(\mathcal{M}_{2}\right)\right)$. Notice that the reason why we have an embedding here is that $\Delta_{\Sigma}^{+}\left(\mathcal{M}_{2}\right)$ is conservative over $\Delta_{\Sigma}^{+}\left(\mathcal{M}_{1}\right)$, meaning that for every $\Sigma^{\left|\mathcal{M}_{1}\right|}$-atom $A$ we have $T \cup \Delta_{\Sigma}^{+}\left(\mathcal{M}_{2}\right) \models A$ iff $T \cup \Delta_{\Sigma}^{+}\left(\mathcal{M}_{1}\right) \models A$ (it is so, because (3) is obtained from the substructure inclusion $\mathcal{M}_{1} \subseteq \mathcal{M}_{2}$ ).

Vice versa, a $T$-presentation of an embedding is a pair of $T$-presentations $\left(X_{1}, \Gamma_{1}\right),\left(X_{2}, \Gamma_{2}\right)$ with $X_{1} \subseteq X_{2}$ and with $\Gamma_{2}$ conservative over $\Gamma_{1}$; to this presentation corresponds the embedding (which we also call canonical)

$F_{T}\left(X_{1}, \Gamma_{1}\right) \longrightarrow F_{T}\left(X_{2}, \Gamma_{2}\right)$ given by the map associating, for every $a \in X_{1}$, the equivalence class of $a$ in $F_{T}\left(X_{1}, \Gamma_{1}\right)$ with the equivalence class of $a$ in $F_{T}\left(X_{2}, \Gamma_{2}\right)$.

Similar considerations apply to $T$-forks: given a $T$-fork $\left(\mathcal{M}_{1}, \mathcal{M}_{2}, \mathcal{A}\right)$, we can associate with it a pair of canonical embeddings among canonical presentations

$$
F_{T}\left(\left|\mathcal{M}_{1}\right|, \Delta_{\Sigma}^{+}\left(\mathcal{M}_{1}\right)\right) \longleftarrow F_{T}\left(|\mathcal{A}|, \Delta_{\Sigma}^{+}(\mathcal{A})\right) \longrightarrow F_{T}\left(\left|\mathcal{M}_{2}\right|, \Delta_{\Sigma}^{+}\left(\mathcal{M}_{2}\right)\right)
$$

A $T$-presentation of a fork is a triple of $T$-presentations $\left(X_{0}, \Gamma_{0}\right),\left(X_{1}, \Gamma_{1}\right),\left(X_{2}, \Gamma_{2}\right)$ with $X_{1} \cap X_{2}=X_{0}, X_{0} \subseteq X_{i}$, and $\Gamma_{i}$ conservative over $\Gamma_{0}(i=1,2)$. To this $T$-presentation it corresponds a pair of canonical embeddings

$$
F_{T}\left(X_{1}, \Gamma_{1}\right) \longleftarrow F_{T}\left(X_{0}, \Gamma_{0}\right) \longrightarrow F_{T}\left(X_{2}, \Gamma_{2}\right)
$$

(which is a $T$-fork, up to renamings). An argument analogous to that used in the proof of Proposition 4.1 shows that the pushout of (4) is given by

$$
F_{T}\left(X_{1}, \Gamma_{1}\right) \stackrel{\mu_{1}}{\longrightarrow} F_{T}\left(X_{1} \cup X_{2}, \Gamma_{1} \cup \Gamma_{2}\right) \stackrel{\mu_{2}}{\longleftarrow} F_{T}\left(X_{2}, \Gamma_{2}\right)
$$

Notice that (for $i=1,2$ ) the map $\mu_{i}$ still associates, for every $a \in X_{i}$, the equivalence class of $a$ in $F_{T}\left(X_{i}, \Gamma_{i}\right)$ with the equivalence class of $a$ in $F_{T}\left(X_{1} \cup X_{2}, \Gamma_{1} \cup \Gamma_{2}\right)$, but this map does not need to be an embedding, because one cannot in general infer that $\Gamma_{1} \cup \Gamma_{2}$ is conservative over $\Gamma_{i}$ from the fact that $\Gamma_{i}$ is conservative over $\Gamma_{0}$ : this is indeed the key property leading to amalgamability (in the case of our Horn theories, if the property fails, amalgamability fails and vice versa).

\subsection{Modularity of Combination Theorem}

In this subsection we will show that Theorem 3.1 allows us to obtain a modular condition of combination. 
Let now $T$ be a $\Sigma$-theory and let $T_{0} \subseteq T$ be a universal Horn $\Sigma_{0}$-theory having the amalgamation property (with $\Sigma_{0} \subseteq \Sigma$ ).

We say that $T$ is $T_{0}$-strongly sub-amalgamable if the sub-amalgamation schema $\sigma_{T_{0}}^{T}$ always contains the minimal $T_{0}$-amalgam (meaning that for every $T$-fork $\left(\mathcal{M}_{1}, \mathcal{M}_{2}, \mathcal{A}\right)$, we have that the minimal $T_{0}$-amalgam of $\left(\mathcal{M}_{1}, \mathcal{M}_{2}, \mathcal{A}\right)$ belongs to $\left.\sigma_{T_{0}}^{T}\left[\left(\mathcal{M}_{1}, \mathcal{M}_{2}, \mathcal{A}\right)\right]\right)$. Notice that, whenever $T_{0}$ is the empty theory in the empty signature, being $T_{0}$-strongly sub-amalgamable is the same as being strongly sub-amalgamable.

Theorem 3.1 immediately implies the following:

Theorem 4.1. If $T_{1}, T_{2}$ are both $T_{0}$-compatible and $T_{0}$-strongly sub-amalgamable (over an amalgamable universal Horn theory $T_{0}$ in their common subsignature $\left.\Sigma_{0}\right)$, then so is $T_{1} \cup T_{2}$.

Proof. Since $T_{1}$ and $T_{2}$ are $T_{0}$-strongly sub-amalgamable, their sub-amalgamation schemata $\sigma_{T_{0}}^{T_{i}}(i=1,2)$ always contain minimal $T_{0}$-amalgams. By Theorem 3.1 ( $T_{1}$ and $T_{2}$ are also $T_{0}$-compatible), this implies that for every $T_{1} \cup T_{2}$-fork $\left(\mathcal{M}_{1}, \mathcal{M}_{2}, \mathcal{A}\right)$, the minimal amalgam $\mathcal{B}$ of $\left(\mathcal{M}_{1}, \mathcal{M}_{2}, \mathcal{A}\right)_{\mid \Sigma_{0}}$ belongs to the set $\sigma_{T_{0}}^{T_{1} \cup T_{2}}\left[\left(\mathcal{M}_{1}, \mathcal{M}_{2}, \mathcal{A}\right)\right]$. Using Proposition 2.1, we conclude that also $T_{1} \cup T_{2}$ is $T_{0}$-compatible and $T_{0}$-strongly sub-amalgamable.

Theorem 4.1 has surprising applications to modal logic. As a first step in this direction, we show that it applies to equational theories with a Boolean reduct.

In the following, we let $B A$ be the theory of Boolean algebras (which is well-known to be amalgamable); we use as signature for Boolean algebras the signature $\Sigma_{B A}$ comprising two unary operations $\cap$ and $\cup$ (for meet and join), the 0-ary operations 0,1 (for zero and one) and a unary operation $\sim$ for complement. We abbreviate $(\sim u) \cup t$ as $u \Rightarrow t ; u_{1} \Leftrightarrow u_{2}$ is defined as $\left(u_{1} \Rightarrow u_{2}\right) \cap\left(u_{2} \Rightarrow u_{1}\right)$. The atom $u \leq t$ is defined as $u \cap t=u$ and $u<t$ stands for $u \leq t \wedge u \neq t$.

A BAO-equational theory ${ }^{5}$ is any theory $T$ whose signature extends the signature of Boolean algebras and whose axioms are all equations and include the Boolean algebra axioms. In Subsection 5.2 below, we shall recall in detail how BAO-equational theories are related to modal propositional logics via Lindenbaum constructions. The fusion of two BAO-equational theories $T_{1}$

\footnotetext{
${ }^{5} \mathrm{BAO}$ stands for 'Boolean algebras with operations'. Notice that we do not assume any normality conditions.
} 
and $T_{2}$ is just their combination $T_{1} \cup T_{2}$ (when speaking of the fusion of $T_{1}$ and $T_{2}$, we assume that $T_{1}$ and $T_{2}$ share only the Boolean algebra operations and no other symbol).

The following Proposition is proved in [11]:

Proposition 4.2. Every BAO-equational theory is BA-compatible.

Proof. Let $T$ be a BAO-equational theory; ${ }^{6}$ the theory of Boolean algebras has as model completion the theory of atomless Boolean algebras [6], ${ }^{7}$ so it is sufficient to show how to embed a model $\mathcal{M}$ of $T$ into a model $\mathcal{M}^{\prime}$ of $T$ which is based on an atomless Boolean algebra. Define a sequence of models of $T$ by: $\mathcal{M}_{0}:=\mathcal{M}, \mathcal{M}_{k+1}:=\mathcal{M}_{k} \times \mathcal{M}_{k}$; define also embeddings $\delta_{k}: \mathcal{M}_{k} \longrightarrow \mathcal{M}_{k+1}$ by $\delta_{k}(a):=\langle a, a\rangle$. Now take as $\mathcal{M}^{\prime}$ the union (more precisely, the inductive limit) of this chain: clearly $\mathcal{M}^{\prime}$ is atomless as a Boolean algebra (no non-zero element is minimal in it, as any $a \in \mathcal{M}_{k}$ gets identified with $\langle a, a\rangle=\langle a, 0\rangle \cup\langle 0, a\rangle$ in $\left.\mathcal{M}_{k+1}\right)$.

As an immediate consequence, from Theorem 4.1, we get:

Corollary 4.1. If two BAO-equational theories $T_{1}$ and $T_{2}$ are both $B A$ strongly amalgamable, so is their fusion.

The next section is devoted to the interpretation of the meaning of the above Corollary in the context of propositional modal logic.

\section{Applications to modal logic}

\subsection{Interpolation in propositional modal logic}

For simplicity, we deal only with unary modalities (and, consequently, we shall consider only BAO-theories whose non-Boolean symbols are unary function symbols), however we point out that the extension to $n$-ary modalities is straightforward.

A modal signature $\Sigma_{M}$ is a set of unary operation symbols; from $\Sigma_{M}$, propositional modal formulae are built using countably many propositional variables, the operation symbols in $\Sigma_{M}$, the Boolean connectives $\cap, \cup, \sim$ and

\footnotetext{
${ }^{6}$ The argument works also for universal Horn theories extending the theory of Boolean algebras.

${ }^{7}$ Recall that a Boolean algebra is atomless iff for every non-zero element $a$ from its support there is a non-zero $b$ such that $b<a$.
} 
the constants 1 for truth and 0 for falsity. We use the letters $x, x_{1}, \ldots, y, y_{1}, \ldots$ to denote propositional variables and the letters $t, t_{1}, \ldots, u, u_{1}, \ldots$ to denote propositional formulae; $t \Rightarrow u$ and $t \Leftrightarrow u$ are abbreviations for $(\sim t) \cup u$ and for $(t \Rightarrow u) \cap(u \Rightarrow t)$, respectively. We use notations like $t(\underline{x})(\operatorname{resp} . \Gamma(\underline{x}))$ to say that the modal formula $t$ (the set of modal formulae $\Gamma$ ) is built up from a set of propositional variables included in the tuple $\underline{x}$.

The following definition is taken from [33], pp. 8-9:

Definition 5.1. A classical modal logic $L$ based on a modal signature $\Sigma_{M}$ is a set of modal formulae that

(i) contains all classical propositional tautologies;

(ii) is closed under uniform substitution of propositional variables by propositional formulae;

(iii) is closed under the modus ponens rule ('from $t$ and $t \Rightarrow u$ infer $u$ ');

(iv) is closed under the replacement rules, which are specified as follows. We have one such rule for each $o \in \Sigma_{M}$, namely:

$$
\frac{t \Leftrightarrow u}{o(t) \Leftrightarrow o(u)}
$$

A classical modal logic $L$ is said to be normal iff for every modal operator $o \in \Sigma_{M}, L$ contains the modal formulae $o(1)$ and $o(y \Rightarrow z) \Rightarrow(o(y) \Rightarrow o(z))$.

Since classical modal logics (based on a given modal signature) are closed under intersections, it makes sense to speak of the least classical modal logic $[S]$ containing a certain set of propositional formulae $S$. If $L=[S]$, we say that $S$ is a set of axiom schemata for $L$.

If $L_{1}$ is a classical modal logic over the modal signature $\Sigma_{M}^{1}$ and $L_{2}$ is a classical modal logic over the modal signature $\Sigma_{M}^{2}$ and $\Sigma_{M}^{1} \cap \Sigma_{M}^{2}=\emptyset$, the fusion $L_{1} \oplus L_{2}$ is the modal logic $\left[L_{1} \cup L_{2}\right]$ over the modal signature $\Sigma_{M}^{1} \cup \Sigma_{M}^{2}$.

Given a modal $\operatorname{logic} L$, a set of modal formulae $\Gamma$ and a modal formula $t$, the global consequence relation $\Gamma \vdash_{L} t$ holds iff there is a finite list of modal formulae $t_{0}, \ldots, t_{n}$ such that: (i) $t_{n}$ is $t$; (ii) each $t_{i}$ is either a member of $L$ or a member of $\Gamma$ or is obtained from previous members of the list by applying one of the two inference rules from Definition 5.1 (i.e. modus ponens and replacement). 
Global consequence relation should be contrasted with local consequence relation, to be indicated with $\vdash_{L} \Gamma \Rightarrow t$ : this holds iff there are $g_{1}, \ldots, g_{n} \in \Gamma$ such that $\bigcap_{i=1}^{n} g_{i} \Rightarrow t$ belongs to $L$. If $\Gamma$ consists of a single modal formula $g$, below we write $g \vdash_{L} t$ and $\vdash_{L} g \Rightarrow t$ instead of $\{g\} \vdash_{L} t$ and of $\vdash_{L}\{g\} \Rightarrow t$.

In case $L$ is normal, one can reduce the global consequence relation to the local one: in fact, it is not difficult to see by induction that the following fact ('deduction theorem') holds:

$$
\Gamma \vdash_{L} t \quad \text { iff } \quad \vdash_{L} o \Gamma \Rightarrow t
$$

where $o \Gamma$ is some finite set of modal formulae (depending on $t$ ) obtained from $\Gamma$ by prefixing a string of modal operators (i.e. elements of $o \Gamma$ are modal formulae of the kind $o_{1}\left(o_{2} \cdots o_{n}(g) \cdots\right)$, for $g \in \Gamma$ and $n \geq 0, o_{1}, \ldots, o_{n} \in$ $\left.\Sigma_{M}\right)$.

Due to the presence of local and global consequence relations, we can formulate two different versions of the Craig's interpolation theorem:

Definition 5.2. Let $L$ be a classical modal logic in a modal signature $\Sigma_{M}$.

(i) We say that $L$ enjoys the local interpolation property iff whenever we have $\vdash_{L} t_{1}(\underline{x}, \underline{y}) \Rightarrow t_{2}(\underline{x}, \underline{z})$ for two modal formulae $t_{1}, t_{2}$, then there is a modal formula $u(\underline{x})$ such that $\vdash_{L} t_{1} \Rightarrow u$ and $\vdash_{L} u \Rightarrow t_{2}$. This is a "implication-based" interpolation property.

(ii) We say that $L$ enjoys the global interpolation property iff whenever we have $t_{1}(\underline{x}, \underline{y}) \vdash_{L} t_{2}(\underline{x}, \underline{z})$ for two modal formulae $t_{1}, t_{2}$, then there is a modal formula $u(\underline{x})$ such that $t_{1} \vdash_{L} u$ and $u \vdash_{L} t_{2}$. This is a "consequence-based" interpolation property.

Related forms of "consequence-based" interpolation (like "deductive interpolation") have been studied in detail in several works [17, 31, 8, 39, 30, $10,9,24,16]$ (see in particular [24] for proofs and references for the connections between deductive interpolation and amalgamation through congruence extension property). For normal modal logics, in view of the above deduction theorem, it is easy to see that the local interpolation property implies the global one (but it is not equivalent to it, see [21]). In the non-normal case, there is no deduction theorem available, so that in order to have an interpolation property encompassing both the local and the global versions, it seems that a different notion needs to be introduced. This is what we are doing now. 
Given a modal logic $L$ and two sets of modal formulae $\Gamma_{1}(\underline{x}, \underline{y}), \Gamma_{2}(\underline{x}, \underline{z})$, let us call an $\underline{x}$-residue chain a tuple of modal formulae $C(\underline{x})=g_{1}(\underline{x}), \ldots, g_{n}(\underline{x})$ such that we have $\Gamma_{1} \cup\left\{g_{1}, \ldots, g_{2 i}\right\} \vdash_{L} g_{2 i+1}$ and $\Gamma_{2} \cup\left\{g_{1}, \ldots, g_{2 j-1}\right\} \vdash_{L} g_{2 j}$, for all $i$ such that $0 \leq 2 i<n$ and for all $j$ such that $0<2 j \leq n$.

Definition 5.3. Let $L$ be a classical modal logic in a modal signature $\Sigma_{M}$.

(iii) We say that $L$ enjoys the comprehensive interpolation property iff whenever we have $\Gamma_{1}(\underline{x}, y), \Gamma_{2}(\underline{x}, \underline{z}) \vdash_{L} t_{1}(\underline{x}, y) \Rightarrow t_{2}(\underline{x}, \underline{z})$ for two modal formulae $t_{1}, t_{2}$ and for two finite sets of modal formulae $\Gamma_{1}, \Gamma_{2}$, there are an $\underline{x}$-residue chain $C(\underline{x})$ and a modal formula $u(\underline{x})$ such that we have $\Gamma_{1}, C \vdash_{L} t_{1} \Rightarrow u$ and $\Gamma_{2}, C \vdash_{L} u \Rightarrow t_{2}$.

Notice that the comprehensive interpolation property implies both the local and the global interpolation properties: the first fact is shown in the proof of Corollary 5.1; the second is stated in the following Proposition:

Proposition 5.1. Let $L$ be a classical modal logic which admits the comprehensive interpolation property. Then, L enjoys the global interpolation property.

Proof. Suppose that $L$ admits the comprehensive interpolation property. In order to show that $L$ is globally interpolating, we consider two modal formulas $\alpha(\underline{x}, \underline{y})$ and $\beta(\underline{x}, \underline{z})$ such that $\alpha \vdash_{L} \beta$. By definition of comprehensive interpolation (defining $\Gamma_{1}:=\alpha, \Gamma_{2}:=\top, t_{1}:=\top$ and $t_{2}:=\beta$ ), there exist an $\underline{x}$-residue chain $C(\underline{x}):=g_{1}(\underline{x}), \ldots, g_{n}(\underline{x})$ and a modal formula $u(\underline{x})$ such that:

- $\Gamma_{1} \cup\left\{g_{1}, \ldots, g_{2 i}\right\} \vdash_{L} g_{2 i+1}$ and $\Gamma_{2} \cup\left\{g_{1}, \ldots, g_{2 j-1}\right\} \vdash_{L} g_{2 j}$, for all $i$ such that $0 \leq 2 i<n$ and for all $j$ such that $0<2 j \leq n$, which implies that $\alpha \vdash_{L} \bigwedge C$.

- $\Gamma_{1}, C \vdash_{L} t_{1} \rightarrow u$ and $\Gamma_{2}, C \vdash_{L} u \rightarrow t_{2}$, which means that $\alpha, C \vdash_{L} u$ and $C \vdash_{L} u \rightarrow \beta$.

Then, it is clear that $\alpha \vdash_{L}(\bigwedge C) \wedge u$ and also $(\bigwedge C) \wedge u \vdash_{L} \beta$. If we define $\theta(\underline{x}):=(\bigwedge C)(\underline{x}) \wedge u(\underline{x})$, we get that $\alpha \vdash_{L} \theta$ and $\theta \vdash_{L} \beta$, as required.

In the normal case, via deduction theorem, it can be easily seen that the comprehensive interpolation property is equivalent to the local interpolation property (Corollary 5.1). 
Remark. The formulation we used for the comprehensive interpolation property is reminiscent of intuitions coming from automated reasoning practice (we suppose we are given reasoners who cooperate by exchanging information over the common sublanguage). It is however possible to give a (trivially) equivalent formulation which is easier to compare with some definitions known from the literature. The equivalent definition is as follows: say that $L$ has the comprehensive interpolation property iff whenever we are given $\Gamma_{1}(\underline{x}, \underline{y}), \Gamma_{2}(\underline{x}, \underline{z})$ - having the property that for every $v(\underline{x})$ we have $\Gamma_{1} \vdash_{L} v$ iff $\Gamma_{2} \vdash_{L} v$ - if it happens that $\Gamma_{1}(\underline{x}, y), \Gamma_{2}(\underline{x}, \underline{z}) \vdash_{L} t_{1}(\underline{x}, y) \Rightarrow t_{2}(\underline{x}, \underline{z})$ for some $t_{1}, t_{2}$, then there is a modal formula $u(\underline{x})$ such that we have $\Gamma_{1} \vdash_{L} t_{1} \Rightarrow u$ and $\Gamma_{2} \vdash_{L} u \Rightarrow t_{2}$. This formulation should be compared e.g. with the (OIP) ('ordinary interpolation property') in [10], Def. 3.3: the comparison clearly shows that our 'comprehensive interpolation property' is a stronger form of (OIP) that includes both local and global aspects (i.e. in the terminology of [10], both 'conditional' and 'deductive' aspects).

Remark. As a further remark, we underline that (as it is not difficult to show), in case the congruence extension property holds, one can assume that all residue chains in the definition of the comprehensive interpolation property have length 1; in this case, again comparing with [10] Def.3.1(iii), our comprehensive interpolation property becomes just a stronger form of the (MIP) ('Maehara interpolation property') comprising both local and global aspects.

Our final result, giving an extension of Wolter's result [38] to non-normal case, is the following:

Theorem 5.1. If the modal logics $L_{1}$ and $L_{2}$ both have the comprehensive interpolation property, so does their fusion $L_{1} \oplus L_{2}$.

The proof of the above Theorem is reported in the final Subsection 5.3; actually, it requires some algebraic logic background which will be introduced in the following subsection.

\subsection{Algebraic logic background}

We now revisit key notions from algebraic logic and recall the bijective correspondence between modal logics and BAO-equational theories [32]. The correspondence works as follows.

Given a logic $L$ with modal signature $\Sigma_{M}$, we define the $B A O$-equational theory $T_{L}$ as the theory having as signature $\Sigma_{L}:=\Sigma_{M} \cup \Sigma_{B A}$ and as set of 
axioms the set

$$
B A \cup\{t=1 \quad \mid t \in L\} .
$$

Notice that, from our notational conventions, it follows that $\Sigma_{M}$-modal formulae are the same as $\Sigma_{L}$-terms. Models of $T_{L}$ will be called L-algebras in the following.

Vice versa, given an equational extension $T$ of $B A$ over the signature $\Sigma$, we define $L_{T}$ as the classical modal logic over the modal signature $\Sigma \backslash \Sigma_{B A}$ axiomatized by the formulae

$$
\{t \mid T \models t=1\} .
$$

Notice that under the above bijection, we have $T_{L_{1} \oplus L_{2}}=T_{L_{1}} \cup T_{L_{2}}$, i.e. the fusion of BAO's (as defined in Subsection 4.2) corresponds to the fusion of modal logics (as defined in Subsection 5.1).

Classical modal logics (in our sense) and equational extensions of $B A$ are equivalent formalisms. For our purposes, it is important to revisit presentations, as introduced in Subsection 4.1, in terms of Lindenbaum-Tarski algebras.

Recall that a $T_{L}$-presentation (we fix a logic $L$ ) is pair $(X, \Gamma)$ given by a set of fresh constants $X$ and a set of $\Sigma_{L}^{X}$-atoms. Now, in the current situation, we can view the $X$ as propositional variables and notice that every atom is equivalent to an atom of the kind $t=1$, where $t$ is a $\Sigma_{L}^{X}$-term (alias modal formula in which at most the $X$ occur). This is so because there are no predicate symbols other than equality in $\Sigma_{L}$ and because we can transform an atom $t_{1}=t_{2}$ into the atom $t_{1} \Leftrightarrow t_{2}=1$, modulo $T_{L}$ equivalence. Thus, from now on, a presentation will be just a pair $(X, \Gamma)$ where the elements of $\Gamma$ are $\Sigma_{L}^{X}$-terms. We recall that the $L$-algebra $F_{T_{L}}(X, \Gamma)$ corresponding to the presentation $(X, \Gamma)$ is built up from equivalence classes of $\Sigma_{L}^{X}$-terms under the equivalence relation $u_{1} \sim_{\Gamma} u_{2}$ given by $T_{L} \cup\{t=1 \mid t \in \Gamma\} \models u_{1}=u_{2}$, by defining all operations on representative elements of equivalence classes.

Now, it is well-known that it is possible to build $F_{T_{L}}(X, \Gamma)$ in another equivalent way (the Lindenbaum-Tarski construction), directly via the global consequence relation $\vdash_{L}$ of the logic $L$. We recall how to do it and show the equivalence with the old construction of $F_{T_{L}}(X, \Gamma)$.

Given a presentation $(X, \Gamma)$, the Lindenbaum-Tarski algebra $L T(X, \Gamma)$ is built as follows: we take the set of the modal formulae containing at most the propositional variables $X$ and introduce the equivalence relation defined by $u_{1} \sim_{\Gamma}^{L T} u_{2}$ iff $\Gamma \vdash_{L} u_{1} \Leftrightarrow u_{2}$. The last equivalence relation is 
a congruence because, for istance, if $u_{1} \sim_{\Gamma}^{L T} u_{2}$ (i.e. $\Gamma \vdash_{L} u_{1} \Leftrightarrow u_{2}$ ), then, applying the replacement rule, we also have $\Gamma \vdash_{L} o\left(u_{1}\right) \Leftrightarrow o\left(u_{2}\right)$, which means $o\left(u_{1}\right) \sim_{\Gamma}^{L T} o\left(u_{2}\right)$. The boolean cases are analogous. Defining each operation on representatives of equivalence classes, we obtain our algebra $\operatorname{LT}(X, \Gamma)$; by construction, $\operatorname{LT}(X, \Gamma)$ is a model of $T_{L}$ and (under the natural evaluation of each variable in $X$ as its own equivalence class) we have that all $u \in \Gamma$ evaluates to 1 .

Proposition 5.2. We have $L T(X, \Gamma) \simeq F_{T_{L}}(X, \Gamma)$, for every presentation $(X, \Gamma)$.

Proof. It is sufficient to observe that (by straightforward arguments) we have

$$
\Gamma \vdash_{L} t \quad \text { iff } \quad T_{L} \cup\{u=1 \mid u \in \Gamma\} \models t=1
$$

for every $t$.

Due to the above Proposition, we shall feel free to use the constructions of $L T(X, \Gamma)$ and of $F_{T_{L}}(X, \Gamma)$ interchangeably.

We dedicate the left subsection of this section to the proof of Theorem 5.1.

\subsection{Proof of Theorem 5.1}

We finally report the proof of our main result from Section 5, namely Theorem 5.1. The claim is to show that the model-theoretic condition of Theorem 4.1, i.e. $T_{0}$-strong sub-amalgamability, in case $T_{0}$ is the theory of Boolean algebras, is nothing but the superamalgamation property known from algebraic logic. Further, we will prove that superamalgamability is equivalent to comprehensive interpolation. By Corollary 4.1, we derive fusion modularity of comprehensive interpolation (and also of local interpolation in the normal case). Before doing that, we recall the definition of superamalgamation.

Definition 5.4. We say that a BAO-equational theory $T$ has the superamalgamation property iff for every $T$-fork $\left(\mathcal{M}_{1}, \mathcal{M}_{2}, \mathcal{A}\right)$ there exists a $T$ amalgam $\left(\mathcal{M}, \mu_{1}, \mu_{2}\right)$ such that for every $a_{1} \in\left|\mathcal{M}_{1}\right|, a_{2} \in\left|\mathcal{M}_{2}\right|$ such that $\mu_{1}\left(a_{1}\right) \leq \mu_{2}\left(a_{2}\right)$ there exists $a_{0} \in|\mathcal{A}|$ such that $a_{1} \leq a_{0}$ holds in $\mathcal{M}_{1}$ and $a_{0} \leq a_{2}$ holds in $\mathcal{M}_{2}$.

We also state the following definition (related to superamalgamation), which involves commutative squares of algebras: 
Definition 5.5. Let $L$ be a classical modal logic. A commutative square of $T_{L}$-algebras

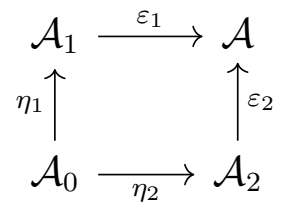

is said to have the interpolation property iff the following holds:

$\forall a_{1} \in\left|\mathcal{A}_{1}\right|, \forall a_{2} \in\left|\mathcal{A}_{2}\right|\left(\varepsilon_{1}\left(a_{1}\right) \leq \varepsilon_{2}\left(a_{2}\right) \Rightarrow \exists b \in\left|\mathcal{A}_{0}\right|\left(a_{1} \leq \eta_{1}(b) \wedge \eta_{2}(b) \leq a_{2}\right)\right)$

Lemma 5.1. In a commutative square having the interpolation property as above, if $\eta_{1}$ is injective (i.e. an embedding), so is $\varepsilon_{2}$.

Proof. Recall that a morphism $\mu$ among Boolean algebras is injective iff $1 \leq \mu(a)$ implies $1 \leq a$ for all $a$. Suppose that $\eta_{1}$ is injective and $1 \leq \varepsilon_{2}(a)$; then $\varepsilon_{1}(1) \leq \varepsilon_{2}(a)$, so there is $b \in\left|\mathcal{A}_{0}\right|$ such that $1 \leq \eta_{1}(b)$ and $\eta_{2}(b) \leq a$; this implies $b=1$ and $1 \leq a$, as required.

Definition 5.6. Let $L$ be a classical modal logic. Given three algebras $\mathcal{A}_{0}$, $\mathcal{A}_{1}$ and $\mathcal{A}_{2}$ endowed with homomorphisms $\eta_{1}: \mathcal{A}_{0} \rightarrow \mathcal{A}_{1}$ and $\eta_{2}: \mathcal{A}_{0} \rightarrow \mathcal{A}_{2}$, we say that they are superamalgamable if there exists another algebra $\mathcal{A}$ with homomorphisms $\varepsilon_{1}: \mathcal{A}_{1} \rightarrow \mathcal{A}$ and $\varepsilon_{2}: \mathcal{A}_{2} \rightarrow \mathcal{A}$ such that the square

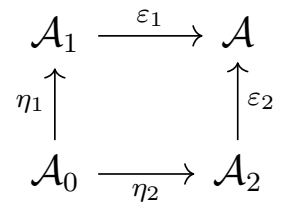

is commutative and has the interpolation property.

Next Proposition slightly restates the superamalgamation property (basically, it shows that in order to check superamalgamation property, we only need to fill a $T_{L}$-fork into a square having the interpolation property, without caring about the fact that the square is formed by embeddings):

Proposition 5.3. The following conditions are equivalent for a modal logic $L$ :

(i) $T_{L}$ has the superamalgamation property; 
(ii) every $T_{L}$-fork is superamalgamable;

(iii) the pushout of every $T_{L}$-fork has the interpolation property.

Proof. The implication (i) $\Rightarrow$ (ii) is trivial, whereas the implication (ii) $\Rightarrow$ (iii) is immediate by the universal property of pushouts. To show that (iii) $\Rightarrow$ (i), assume (iii) and take a $T_{L}$-fork $\left(\mathcal{A}_{1}, \mathcal{A}_{2}, \mathcal{A}_{0}\right)$; the related pushout

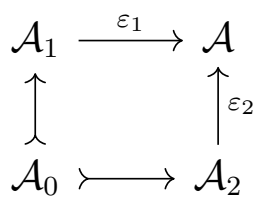

has the interpolation property and by Lemma 5.1 , it follows that $\varepsilon_{2}$ is injective. Exchanging $\mathcal{A}_{1}$ and $\mathcal{A}_{2}$ (i.e. considering the fork $\left(\mathcal{A}_{2}, \mathcal{A}_{1}, \mathcal{A}_{0}\right)$ ), it follows that $\varepsilon_{1}$ is also injective (the pushout construction is symmetric). Thus, we have found a $T$-amalgam of the fork having the interpolation property.

Notice that, up to a renaming isomorphism, every triple $\mathcal{A}_{0}, \mathcal{A}_{1}$ and $\mathcal{A}_{2}$ of $L$-algebras endowed with embeddings $\eta_{1}: \mathcal{A}_{0} \longmapsto \mathcal{A}_{1}$ and $\eta_{2}: \mathcal{A}_{0} \longmapsto \mathcal{A}_{2}$ is a $T_{L}$-fork; as a consequence, $T_{L}$ is superamalgamable iff every such a triple of L-algebras connected by embeddings is superamalgamable.

The following proposition relates the comprehensive interpolation property (which is a linguistic notion for propositional modal logics) to superamalgamability (which is an algebraic condition):

Proposition 5.4. Let $L$ be a modal logic. Then, $T_{L}$ has the superamalgamability property iff $L$ satisfies the comprehensive interpolation property.

Proof. Suppose that $L$ has the comprehensive interpolation property. Consider a $T_{L}$-fork; using presentations, we can suppose that the fork is given by a triple of $T_{L}$-presentations $\left(X_{0}, \Gamma_{0}\right),\left(X_{0} \cup X_{1}, \Gamma_{1}\right),\left(X_{0} \cup X_{2}, \Gamma_{2}\right)$ with $\left(X_{0} \cup X_{1}\right) \cap\left(X_{0} \cup X_{2}\right)=X_{0}$, and with $\Gamma_{i}$ conservative over $\Gamma_{0}(i=1,2)$. To this $T_{L}$-presentation corresponds a pair of canonical embeddings (let us write $X_{0}, X_{i}$ instead of $X_{0} \cup X_{i}$ for simplicity):

$$
F_{T_{L}}\left(X_{0}, X_{1}, \Gamma_{1}\right) \stackrel{\eta_{1}}{\longleftarrow} F_{T_{L}}\left(X_{0}, \Gamma_{0}\right) \stackrel{\eta_{2}}{\longrightarrow} F_{T_{L}}\left(X_{0}, X_{2}, \Gamma_{2}\right)
$$

(we recall that we use the word 'canonical' to mean that $\eta_{i}$ associates the equivalence class of $t$ in $F_{T_{L}}\left(X_{0}, \Gamma_{0}\right)$ with the equivalence class of the same 
$t$ in $\left.F_{T_{L}}\left(X_{0}, X_{i}, \Gamma_{i}\right)\right) .{ }^{8}$ We are using the Lindenbaum-Tarski construction for presentations, hence conservativity of $\Gamma_{i}$ means that we have

$$
\Gamma_{i} \vdash_{L} t\left(\underline{x}_{0}\right) \quad \text { iff } \quad \Gamma_{0} \vdash_{L} t\left(\underline{x}_{0}\right)
$$

for every modal formula (alias $\Sigma_{L}$-term) containing at most propositional variables $\underline{x}_{0} \subseteq X_{0}$.

From (5), we know that the pushout of (6) is given by

$$
F_{T_{L}}\left(X_{0}, X_{1}, \Gamma_{1}\right) \stackrel{\varepsilon_{1}}{\longrightarrow} F_{T_{L}}\left(X_{0}, X_{1}, X_{2}, \Gamma_{1} \cup \Gamma_{2}\right) \stackrel{\varepsilon_{2}}{\longleftarrow} F_{T_{L}}\left(X_{0}, X_{2}, \Gamma_{2}\right)
$$

with canonical maps $\varepsilon_{1}, \varepsilon_{2}$.

We use Proposition 5.3 and just show that the square formed by the quadruple $\left(\eta_{1}, \eta_{2}, \varepsilon_{1}, \varepsilon_{2}\right)$ has the interpolation property. To this aim, let us suppose that for modal formulae $t_{1}, t_{2}$ (where $t_{1}$ is built up from the variables in $X_{0} \cup X_{1}$ and $t_{2}$ is built up from the variables in $X_{0} \cup X_{2}$ ) we have

$$
\varepsilon_{1}\left(\left[t_{1}\right]\right)=\left[t_{1}\right] \leq\left[t_{2}\right]=\varepsilon_{2}\left(\left[t_{2}\right]\right)
$$

in $F_{T_{L}}\left(X_{0}, X_{1}, X_{2}, \Gamma_{1} \cup \Gamma_{2}\right)$; the last means that $\Gamma_{1} \cup \Gamma_{2} \vdash_{L} t_{1} \Rightarrow t_{2}$ by the construction of the Lindenbaum-Tarski algebra. Since only finitely many formulae are used in a derivation, there exist two finite subsets $\Gamma_{1}^{\prime} \subseteq \Gamma_{1}$ and $\Gamma_{2}^{\prime} \subseteq \Gamma_{2}$ such that

$$
\Gamma_{1}^{\prime}\left(\underline{x}_{0}, \underline{x}_{1}\right), \Gamma_{2}^{\prime}\left(\underline{x}_{0}, \underline{x}_{2}\right) \vdash_{L} t_{1}\left(\underline{x}_{0}, \underline{x}_{1}\right) \Rightarrow t_{2}\left(\underline{x}_{0}, \underline{x}_{2}\right)
$$

(here $\underline{x}_{0}$ is a tuple including all the variables from $X_{0}$ occurring in $\Gamma_{1}^{\prime}, \Gamma_{2}^{\prime}, t_{1}, t_{2}$, $\underline{x}_{1}$ is a tuple including all the variables from $X_{1}$ occurring in $\Gamma_{1}^{\prime}, t_{1}$ and $\underline{x}_{2}$ is a tuple including all the variables from $X_{2}$ occurring in $\Gamma_{2}^{\prime}, t_{2}$ ). By the comprehensive interpolation property, there exist a formula $u\left(\underline{x}_{0}\right)$ and a finite set of formulae $g_{1}\left(\underline{x}_{0}\right), \ldots, g_{n}\left(\underline{x}_{0}\right)$ such that:

$$
\begin{gathered}
\Gamma_{1}^{\prime}\left(\underline{x}_{0}, \underline{x}_{1}\right) \vdash_{L} g_{1} \\
\Gamma_{2}^{\prime}\left(\underline{x}_{0}, \underline{x}_{2}\right), g_{1} \vdash_{L} g_{2}
\end{gathered}
$$

\footnotetext{
${ }^{8}$ Notationally, the fact that $\eta_{i}$ is canonical allows us to write equations like $\eta_{i}([t])=[t]$; there is a bit of abuse in this notation, because we do not indicate where the equivalence class $[t]$ of $t$ is taken from, but such missed information can be easily deduced from the context.
} 


$$
\begin{aligned}
& \Gamma_{1}^{\prime}\left(\underline{x}_{0}, \underline{x}_{1}\right), g_{1}, \ldots, g_{2 j-1} \vdash_{L} g_{2 j} \\
& \Gamma_{2}^{\prime}\left(\underline{x}_{0}, \underline{x}_{2}\right), g_{1}, \ldots, g_{2 k} \vdash_{L} g_{2 k+1}
\end{aligned}
$$

and also such that:

$$
\begin{aligned}
& \Gamma_{1}^{\prime}\left(\underline{x}_{0}, \underline{x}_{1}\right), g_{1}, \ldots, g_{n} \vdash_{L} t_{1} \Rightarrow u \\
& \Gamma_{2}^{\prime}\left(\underline{x}_{0}, \underline{x}_{2}\right), g_{1}, \ldots, g_{n} \vdash_{L} u \Rightarrow t_{2}
\end{aligned}
$$

But, for $i=1,2$, by conservativity of $\Gamma_{i}$ over $\Gamma_{0}$, we obtain that $\Gamma_{i} \vdash_{L} g_{j}$ (all $j=1, \ldots, n)$, hence $\Gamma_{1} \vdash_{L} t_{1} \Rightarrow u$ and $\Gamma_{2} \vdash_{L} u \Rightarrow t_{2}$. The last two facts yield $\left[t_{1}\right] \leq[u]=\eta_{1}([u])$ in $F_{T_{L}}\left(X_{0}, X_{1}, \Gamma_{1}\right)$ and $[u]=\eta_{2}([u]) \leq\left[t_{2}\right]$ in $F_{T_{L}}\left(X_{0}, X_{2}, \Gamma_{2}\right)$, as required for the interpolation property to hold for the square $\left(\eta_{1}, \eta_{2}, \varepsilon_{1}, \varepsilon_{2}\right)$.

Conversely, suppose that $L$ has the superamalgamability property. Let $\Gamma_{1}(\underline{x}, \underline{y}), \Gamma_{2}(\underline{x}, \underline{z})$ be finite sets of modal formulae and let $t_{1}(\underline{x}, \underline{y}), t_{2}(\underline{x}, \underline{z})$ be such that $\Gamma_{1}(\underline{x}, \underline{y}), \Gamma_{2}(\underline{x}, \underline{z}) \vdash_{L} t_{1}(\underline{x}, \underline{y}) \Rightarrow t_{2}(\underline{x}, \underline{z})$. We construct three algebras in $T_{L}$ connected with (canonical) monomorphisms in order to apply the superamalgamability property. Let $\Gamma_{0}(\underline{x})$ be the set of the modal formulae $g(\underline{x})$ such that there is an $\underline{x}$-residue chain from $\Gamma_{1}(\underline{x}, y), \Gamma_{2}(\underline{x}, \underline{z})$ ending up in $g(\underline{x})$. Then, we put $\Delta_{1}:=\Gamma_{1} \cup \Gamma_{0}$ and $\Delta_{2}:=\Gamma_{2} \cup \bar{\Gamma}_{0}$; clearly $\Delta_{1}$ and $\Delta_{2}$ are conservative over $\Gamma_{0}$ by construction. Now, we are ready to take:

$$
\begin{aligned}
\mathcal{A}_{1} & :=F_{T_{L}}\left(\underline{x}, \underline{y}, \Delta_{1}\right) \\
\mathcal{A}_{2} & :=F_{T_{L}}\left(\underline{x}, \underline{z}, \Delta_{2}\right) \\
\mathcal{A}_{0} & :=F_{T_{L}}\left(\underline{x}, \Gamma_{0}\right)
\end{aligned}
$$

This is a triple of $L$-agebras connected by canonical embeddings. Then, by the superamalgamability property and by Proposition 5.3, the related pushout has the interpolation property. From (5), we know that such pushout is

$$
F_{T_{L}}\left(\underline{x}, \underline{y}, \Delta_{1}\right) \stackrel{\varepsilon_{1}}{\longrightarrow} F_{T_{L}}\left(\underline{x}, \underline{y}, \underline{z}, \Delta_{1} \cup \Delta_{2}\right) \stackrel{\varepsilon_{2}}{\longleftarrow} F_{T_{L}}\left(\underline{x}, \underline{z}, \Delta_{2}\right)
$$

with canonical maps $\varepsilon_{1}, \varepsilon_{2}$.

From $\Gamma_{1}(\underline{x}, \underline{y}), \Gamma_{2}(\underline{x}, \underline{z}) \vdash_{L} t_{1}(\underline{x}, \underline{y}) \Rightarrow t_{2}(\underline{x}, \underline{z})$, it follows that we have

$$
\varepsilon_{1}\left(\left[t_{1}(\underline{x}, \underline{y})\right]\right)=\left[t_{1}(\underline{x}, \underline{y})\right] \leq\left[t_{2}(\underline{x}, \underline{z})\right]=\varepsilon_{2}\left(\left[t_{2}(\underline{x}, \underline{z})\right]\right)
$$


in $F_{T_{L}}\left(\underline{x}, \underline{y}, \underline{z}, \Delta_{1} \cup \Delta_{2}\right)$. By the interpolation property of the square, there exists a formula $u(\underline{x})$ such that we have $\left[t_{1}\right] \leq[u]=\eta_{1}[u]$ in $F_{T_{L}}\left(\underline{x}, y, \Delta_{1}\right)$ and $\eta_{2}([u])=[u] \leq\left[t_{2}\right]$ in $F_{T_{L}}\left(\underline{x}, \underline{z}, \Delta_{2}\right)$. This means that we get $\Gamma_{1}, \Gamma_{0} F_{L} t_{1} \Rightarrow u$ and $\Gamma_{2}, \Gamma_{0} \vdash_{L} u \Rightarrow t_{2}$. Since only finitely many modal formulae from $\Gamma_{0}$ are involved in these derivations and since all modal formulae in $\Gamma_{0}$ are obtained via $\underline{x}$-residue chains, the claim follows (we can obviously glue finitely many $\underline{x}$-residue chains into a single one).

The last statement of the following Corollary is well-known ([21],[13],[19]):

Corollary 5.1. If $L$ is normal, then $L$ has the comprehensive interpolation property iff it has the local interpolation property. Consequently $T_{L}$ has the superamalgamability property iff $L$ satisfies the local interpolation property.

Proof. Suppose first that $L$ has the local interpolation property. Consider the deduction relation $\Gamma_{1}(\underline{x}, y), \Gamma_{2}(\underline{x}, \underline{z}) \vdash_{L} t_{1}(\underline{x}, y) \Rightarrow t_{2}(\underline{x}, \underline{z})$. Since $L$ is normal, applying the 'deduction theorem' it is clear that the last fact is equivalent to ${ }^{9}$ $\vdash_{L}\left(o \Gamma_{1} \cap o \Gamma_{2}\right) \Rightarrow\left(t_{1} \Rightarrow t_{2}\right)$, which implies (using propositional tautologies) that $\vdash_{L}\left(o \Gamma_{1} \cap t_{1}\right) \Rightarrow\left(o \Gamma_{2} \Rightarrow t_{2}\right)$ holds. By the local interpolation property, there exists a formula $u(\underline{x})$ such that $\vdash_{L}\left(o \Gamma_{1} \cap t_{1}\right) \Rightarrow u$ and $\vdash_{L} u \Rightarrow\left(o \Gamma_{2} \Rightarrow\right.$ $\left.t_{2}\right)$. Applying again propositional tautologies and the 'deduction theorem', the previous statement is equivalent to $\Gamma_{1} \vdash_{L} t_{1} \Rightarrow u$ and $\Gamma_{2} \vdash_{L} u \Rightarrow t_{2}$, which means that $L$ has the comprehensive interpolation property (here, the $\underline{x}$-residue chain is empty).

For the other implication ${ }^{10}$, suppose that $L$ has the comprehensive interpolation property and that $t_{2}(\underline{x}, \underline{z})$ is locally deducible from $t_{1}(\underline{x}, \underline{y})$, i.e. that $\vdash_{L} t_{1}(\underline{x}, y) \Rightarrow t_{2}(\underline{x}, \underline{z})$ holds. This fact implies, by comprehensive interpolation property, that there exist a $\underline{x}$-residue chain $C=\left\{g_{1}, \ldots, g_{n}\right\}$ and a formula $u(\underline{x})$ such that $C \vdash_{L} t_{1} \Rightarrow u$ and $C \vdash_{L} u \Rightarrow t_{2}$. Hence, in order to achieve the aim it is sufficient to show that $\vdash_{L} C$ holds. In fact, since $\Gamma_{i}=\emptyset$, we have $\vdash_{L} g_{1}$; moreover, reasoning by induction, $\vdash_{L} g_{1}, \ldots, \vdash_{L} g_{n-1}$ and $g_{1}, \ldots, g_{n-1} \vdash_{L} g_{n}$ imply, replacing the $g_{i}(i=1, \ldots, n-1)$ with their proofs, that $\vdash_{L} g_{n}$. Therefore, we conclude $\vdash_{L} C$, as wanted.

\footnotetext{
${ }^{9}$ Recall from Subsection 5.1, that we use the notation $o \Gamma$ to indicate the conjunction of a finite set of modal formulae of the kind $o_{1}\left(o_{2} \cdots o_{n}(g) \cdots\right)$, for $g \in \Gamma$ and $n \geq 0$, $o_{1}, \ldots, o_{n} \in \Sigma_{M}$.

${ }^{10}$ Notice that the following argument does not require the normality of $L$. Thus, the comprehensive interpolation property implies the local interpolation property even in the non-normal case.
} 
Classical propositional logic can be seen as the modal logic over the empty signature (as such, it is clearly also normal and Corollary 5.1 applies to it). From ordinary Craig interpolation theorem for classical propositional logic and Corollary 5.1, we can get the following very well-known fact:

Lemma 5.2. BA has the superamalgamation property.

Pushouts in $B A$ can be better described:

Lemma 5.3. Let $\left(\mathcal{A}_{1}, \mathcal{A}_{2}, \mathcal{A}_{0}\right)$ be a $B$ A-fork and let

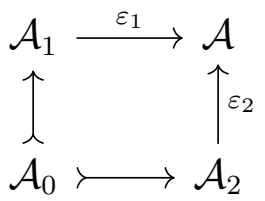

be the Boolean pushout of the fork; then all elements of $|\mathcal{A}|$ can be written as finite meets of elements of the kind $\varepsilon_{1}\left(a_{1}\right) \Rightarrow \varepsilon_{2}\left(a_{2}\right)$, for $a_{1} \in\left|\mathcal{A}_{1}\right|$ and $a_{2} \in\left|\mathcal{A}_{2}\right|$.

Proof. We replace our $B A$-fork with a canonical presentation

$$
F_{B A}\left(X_{0}, X_{1}, \Gamma_{1}\right) \stackrel{\eta_{1}}{\longleftarrow} F_{B A}\left(X_{0}, \Gamma_{0}\right) \stackrel{\eta_{2}}{\longrightarrow} F_{B A}\left(X_{0}, X_{2}, \Gamma_{2}\right)
$$

of it; then the pushout can be presented as

$$
F_{B A}\left(X_{0}, X_{1}, \Gamma_{1}\right) \stackrel{\varepsilon_{1}}{\longrightarrow} F_{B A}\left(X_{0}, X_{1}, X_{2}, \Gamma_{1} \cup \Gamma_{2}\right) \stackrel{\varepsilon_{2}}{\longleftarrow} F_{B A}\left(X_{0}, X_{2}, \Gamma_{2}\right)
$$

Elements of $F_{B A}\left(X_{0}, X_{1}, X_{2}, \Gamma_{1} \cup \Gamma_{2}\right)$ are equivalence classes of classical propositional formulae built up from the variables $X_{0}, X_{1}, X_{2}$. By conjunctive normal forms, they are conjunctions of clauses $l_{1}^{1} \vee \cdots \vee l_{n}^{1} \vee l_{1}^{2} \vee \cdots \vee l_{m}^{2}$, where the literals $l_{j}^{1}$ are built from $X_{0} \cup X_{1}$ and the literals $l_{k}^{2}$ are built up from $X_{0} \cup X_{2}$ (this representation is of course not unique). These clauses in turn can be written as

$$
\left(\neg l_{1}^{1} \wedge \cdots \wedge \neg l_{n}^{1}\right) \Rightarrow\left(l_{1}^{2} \vee \cdots \vee l_{m}^{2}\right)
$$

and the equivalence class of (10) in $F_{B A}\left(X_{0}, X_{1}, X_{2}, \Gamma_{1} \cup \Gamma_{2}\right)$ is of the desired shape.

We can now state the second main result of this paper: 
Theorem 5.2. A BAO-equational theory $T$ has the superamalgamation property iff it is BA-strongly amalgamable.

Proof. Let $T$ be equal to $T_{L}$ for a modal logic $L$.

Suppose first that $T_{L}$ has the superamalgamation property and consider a $T_{L}$-fork $\left(\mathcal{A}_{1}, \mathcal{A}_{2}, \mathcal{A}_{0}\right)$. Since $T_{L}$ is superamalgamable, we can fill the inclusions $\mathcal{A}_{0} \subseteq \mathcal{A}_{1}$ and $\mathcal{A}_{0} \subseteq \mathcal{A}_{2}$ into a commutative square

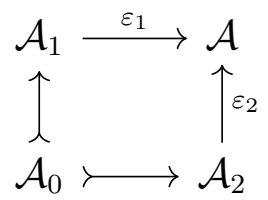

having the interpolation property. Considering the $B A$-reducts, we can build the Boolean pushout square and the Boolean comparison morphism $\theta$ as in the following diagram:

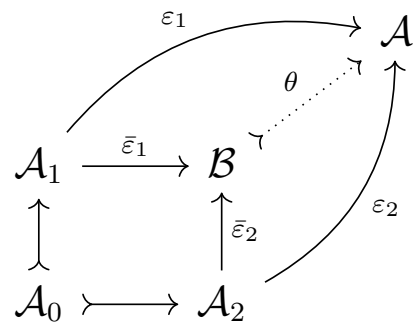

We show that $\theta$ is injective (i.e. a Boolean embedding): this is precisely what it is required by $B A$-strong amalgamability. Using Lemma 5.3 (and recalling that in a Boolean algebra the meet of a finite set is equal to 1 iff all elements from the set are equal to 1$)$, it is sufficient to prove that if $\theta\left(\bar{\varepsilon}_{1}\left(a_{1}\right) \Rightarrow \bar{\varepsilon}_{2}\left(a_{2}\right)\right)=1$, then $\bar{\varepsilon}_{1}\left(a_{1}\right) \leq \bar{\varepsilon}_{2}\left(a_{2}\right)$ (i.e. $\left.\bar{\varepsilon}_{1}\left(a_{1}\right) \Rightarrow \bar{\varepsilon}_{2}\left(a_{2}\right)=1\right)$. But

$$
1=\theta\left(\bar{\varepsilon}_{1}\left(a_{1}\right) \Rightarrow \bar{\varepsilon}_{2}\left(a_{2}\right)\right)=\theta\left(\bar{\varepsilon}_{1}\left(a_{1}\right)\right) \Rightarrow \theta\left(\bar{\varepsilon}_{2}\left(a_{2}\right)\right)=\varepsilon_{1}\left(a_{1}\right) \Rightarrow \varepsilon_{2}\left(a_{2}\right)
$$

and in a Boolean algebra, this is equivalent to $\varepsilon_{1}\left(a_{1}\right) \leq \varepsilon_{2}\left(a_{2}\right)$. Since the outer square has the interpolation property, we conclude that there exists an element $a \in\left|\mathcal{A}_{0}\right|$ such that $a_{1} \leq \eta_{1}(a)$ and $\eta_{2}(a) \leq a_{2}$, where $\eta_{1}, \eta_{2}$ are the inclusions of $\mathcal{A}_{0}$ into $\mathcal{A}_{1}, \mathcal{A}_{2}$. Therefore, we compute $\bar{\varepsilon}_{1}\left(a_{1}\right) \leq \bar{\varepsilon}_{1}\left(\eta_{1}(a)\right)=$ $\bar{\varepsilon}_{2}\left(\eta_{2}(a)\right) \leq \bar{\varepsilon}_{2}\left(a_{2}\right)$, as wanted.

Conversely, suppose that $T_{L}$ is $B A$-strongly amalgamable. Thus, by Proposition 5.3, it is sufficient to show that every $T_{L}$-fork $\left(\mathcal{A}_{1}, \mathcal{A}_{2}, \mathcal{A}_{0}\right)$ is superamalgamable. By $B A$-strong amalgamability, the minimal $B A$-amalgam 
$\left(\mathcal{B}, \bar{\varepsilon}_{1}, \bar{\varepsilon}_{2}\right)$, which is the pushout in $B A$, can be embedded via a $\theta$ (unique by the universal property of the pushout) into a $T_{L}$-amalgam $\left(\mathcal{A}, \varepsilon_{1}, \varepsilon_{2}\right)$.

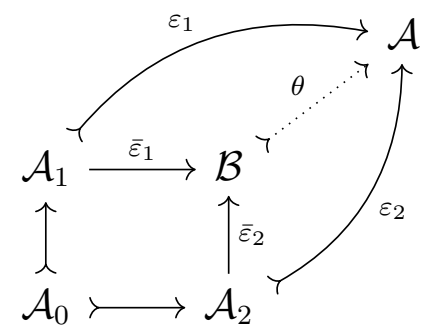

Now Lemma 5.2 states that $B A$ has the superamalgamation property; so, by Proposition 5.3, the Boolean pushout

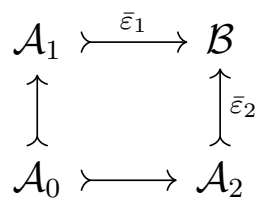

has the interpolation property. We show that, since $\theta$ is injective, the commutative square

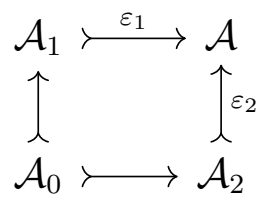

has the interpolation property too. In fact, if, for $a_{1} \in\left|\mathcal{A}_{1}\right|$ and $a_{2} \in\left|\mathcal{A}_{2}\right|$, we have $\varepsilon_{1}\left(a_{1}\right) \leq \varepsilon_{2}\left(a_{2}\right)$ in $\mathcal{A}$, then we get $\theta\left(\bar{\varepsilon}_{1}\left(a_{1}\right)\right) \leq \theta\left(\bar{\varepsilon}_{2}\left(a_{2}\right)\right)$, and also $\bar{\varepsilon}_{1}\left(a_{1}\right) \leq \bar{\varepsilon}_{2}\left(a_{2}\right)$, because $\theta$ is injective. Thus, by the fact that the Boolean pushout has the interpolation property, we conclude that:

$$
\exists a_{0} \in\left|\mathcal{A}_{0}\right|\left(a_{1} \leq \eta_{1}\left(a_{0}\right) \wedge \eta_{2}\left(a_{0}\right) \leq a_{2}\right)
$$

where $\eta_{1}, \eta_{2}$ are the inclusions of $\mathcal{A}_{0}$ into $\mathcal{A}_{1}, \mathcal{A}_{2}$, as wanted.

As an immediate consequence, from Corollary 4.1, we get fusion modularity of superamalgamation:

Corollary 5.2. If two BAO-equational theories $T_{1}$ and $T_{2}$ both have the superamalgamability property, so does their fusion. 
Now, we are ready for our final main result:

Theorem 5.1 If the modal logics $L_{1}$ and $L_{2}$ both have the comprehensive interpolation property, so does their fusion $L_{1} \oplus L_{2}$.

Proof. Immediate, from Proposition 5.4 and Corollary 5.2.

Finally, we state Wolter's theorem [38], which is an immediate consequence of Theorem 5.1 and of Corollary 5.1:

Theorem 5.3. If the normal modal logics $L_{1}$ and $L_{2}$ both have the local interpolation property, so does their fusion $L_{1} \oplus L_{2}$.

\section{Conclusions and future work}

In this paper we considered the problem of transferring interpolation properties to fusions of (non normal) modal logics. We attacked the problem not via specific modal logic techniques, but via general tools applying to combined first-order theories.

Our approach is characterized by the attempt of employing concepts of general scope in several different areas of mathematical logic: in this paper, in particular, model-theoretic notions are shown to be powerful enough to solve seemingly unrelated problems coming from non-classical propositional logics. Algebraic logic, in this way, becomes the powerful tool revealing an unexpected connection to the first-order framework.

We first obtained a sufficient condition (Theorem 3.1) in terms of subamalgamation schemata, allowing to transfer quantifier-free interpolation property from two theories to their union, in the case where the two theories share symbols other than pure equality. We are not aware of previous papers attacking this problem (one should however mention a series of papers $[34,35,37]$ analyzing the somewhat related - but different - problem of transferring, in a hierarchical way, interpolation properties to theory extensions under 'locality' assumptions). The sufficient condition of Theorem 3.1 was used to get a modular condition in case the shared theory is universal Horn (Theorem 4.1). For equational theories extending the theory of Boolean algebras, this modular condition turns out to be equivalent to the superamalgamability condition known from algebraic logic [22]. Thus, our results immediately imply the fusion transfer of local interpolation property [38] for classical normal modal logics. In the general non-normal case, the modularity of superamalgamability can be translated into a fusion transfer result 
for a new kind of interpolation property (which we called 'comprehensive interpolation property').

Still, many problems need to be faced by future research. Our combinability conditions should be characterizable from a syntactic point of view and, from such syntactic characterizations, we expect to be able to design concrete combined interpolation algorithms. Concerning modal logic, besides the old question about modularity of local interpolation property in the non-normal case, new questions arise concerning the status of the new comprehensive interpolation property: is it really stronger than other forms of interpolation property (e.g. than the local one)? Are there different ways of specifying it? Is it modular also for modal logics on a non-classical basis?

Acknowledgements. The first author was supported by the GNSAGA group of INdAM (Istituto Nazionale di Alta Matematica).

\section{References}

[1] Adámek, J., \& Rosický, J. (1994). Locally presentable and accessible categories volume 189 of London Mathematical Society Lecture Note Series. Cambridge University Press, Cambridge.

[2] Baader, F., Ghilardi, S., \& Tinelli, C. (2006). A new combination procedure for the word problem that generalizes fusion decidability results in modal logics. Inf. Comput., 204, 1413-1452.

[3] Bacsich, P. D. (1975). Amalgamation properties and interpolation theorems for equational theories. Algebra Universalis, 5, 45-55.

[4] Bruttomesso, R., Ghilardi, S., \& Ranise, S. (2012). From strong amalgamability to modularity of quantifier-free interpolation. In Proc. of IJCAR 2012 (pp. 118-133).

[5] Bruttomesso, R., Ghilardi, S., \& Ranise, S. (2014). Quantifier-free interpolation in combinations of equality interpolating theories. ACM Trans. Comput. Log., 15, 5:1-5:34.

[6] Chang, C.-C., \& Keisler, J. H. (1990). Model Theory. (3rd ed.). Amsterdam-London: North-Holland.

[7] Craig, W. (1957). Three uses of the Herbrand-Gentzen theorem in relating model theory and proof theory. J. Symb. Log., (pp. 269-285). 
[8] Czelakowski, J. (1985). Sentential logics and Maehara interpolation property. Studia Logica, 44, 265-283.

[9] Czelakowski, J. (2007). Fregean logics and the strong amalgamation property. Bull. Sect. Logic, 26, 105-116.

[10] Czelakowski, J., \& Pigozzi, D. (1999). Amalgamation and interpolation in abstract algebraic logic. In Models, Algebras, and Proofs. CRC Press volume 203 of Lecture Notes in Pure and Applied Mathematics.

[11] Ghilardi, S. (2004). Model theoretic methods in combined constraint satisfiability. Journal of Automated Reasoning, 33, 221-249.

[12] Ghilardi, S., \& Gianola, A. (2017). Interpolation, Amalgamation and Combination (The Non-disjoint Signatures Case). In Proc. of FroCoS 2017 (pp. 316-332). Springer LNAI.

[13] Ghilardi, S., \& Meloni, G. C. (1990). Modal logics with $n$-ary connectives. Z. Math. Logik Grundlag. Math., 36, 193-215.

[14] Ghilardi, S., Nicolini, E., \& Zucchelli, D. (2008). A comprehensive framework for combined decision procedures. ACM Transactions on Computational Logic, 9, 1-54.

[15] Gianola, A. (2017). Interpolation, Amalgamation and Combination. Master Thesis. Università degli Studi di Milano.

[16] van Gool, S., Metcalfe, G., \& Tsinakis, C. (To appear). Uniform interpolation and compact congruences. Ann. Pure Appl. Logic, .

[17] Jónsson, B. (1965). Extensions of relational structures. Proc. International Symposium on the Theory of Models, Berkeley, (pp. 146-157).

[18] Kiss, E., Marki, L., Prohle, P., \& Tholen, W. (1983). Categorical algebraic properties. a compendium on amalgamation, congruence extension, epimorphisms, residual smallness, and injectivity. Studia Sci. Math. Hungar., 18, 79-141.

[19] Kracht, M. (2007). Modal consequence relations. Handbook of Modal Logic, 3, 491 - 545. 
[20] Maksimova, L. L. (1977). Craig's theorem in superintuitionistic logics and amalgamable varieties. Algebra $i$ Logika, 16, 643-681, 741.

[21] Maksimova, L. L. (1979). Interpolation theorems in modal logics and amalgamable varieties of topological Boolean algebras. Algebra $i$ Logika, $18,556-586,632$.

[22] Maksimova, L. L. (1980). Interpolation theorems in modal logics. Sufficient conditions. Algebra i Logika, 19, 194-213, 250-251.

[23] McMillan, K. L. (2005). Applications of Craig Interpolation to Model Checking. In Proc. of TACAS LNCS (pp. 1-12).

[24] Metcalfe, G., Montagna, F., \& Tsinakis, C. (2014). Amalgamation and interpolation in ordered algebras. J. Algebra, 402, 21-82.

[25] Nelson, G., \& Oppen, D. C. (1979). Simplification by cooperating decision procedures. ACM Transaction on Programming Languages and Systems, 1, 245-257.

[26] Nicolini, E., Ringeissen, C., \& Rusinowitch, M. (2009). Combinable extensions of abelian groups. In Proc. of CADE-22 (pp. 51-66).

[27] Nicolini, E., Ringeissen, C., \& Rusinowitch, M. (2009). Data structures with arithmetic constraints: A non-disjoint combination. In Proc. of FroCoS 2009 (pp. 319-334).

[28] Nicolini, E., Ringeissen, C., \& Rusinowitch, M. (2009). Satisfiability procedures for combination of theories sharing integer offsets. In Proc. of TACAS 2009 (pp. 428-442).

[29] Nicolini, E., Ringeissen, C., \& Rusinowitch, M. (2010). Combining satisfiability procedures for unions of theories with a shared counting operator. Fundam. Inform., 105, 163-187.

[30] Ono, H. (1986). Interpolation and the Robinson property for logics not closed under the Boolean operations. Algebra Universalis, 23, 111-122.

[31] Pigozzi, D. (1972). Amalgamations, congruence-extension, and interpolation properties in algebras. Algebra Universalis, 1, 269-349. 
[32] Rasiowa, H. (1974). An algebraic approach to non-classical logics. NorthHolland Publishing Co., Amsterdam-London; American Elsevier Publishing Co., Inc., New York. Studies in Logic and the Foundations of Mathematics, Vol. 78.

[33] Segerberg, K. (1971). An Essay in Classical Modal Logic volume 13 of Filosofiska Studier. Uppsala Universitet.

[34] Sofronie-Stokkermans, V. (2008). Interpolation in local theory extensions. Logical Methods in Computer Science, 4.

[35] Sofronie-Stokkermans, V. (2016). On interpolation and symbol elimination in theory extensions. In Proc. of IJCAR 2016 (pp. 273-289).

[36] Tinelli, C., \& Harandi, M. T. (1996). A new correctness proof of the Nelson-Oppen combination procedure. In Proc. of FroCoS 1996 Applied Logic (pp. 103-120). Kluwer Academic Publishers.

[37] Totla, N., \& Wies, T. (2016). Complete instantiation-based interpolation. J. Autom. Reasoning, 57, 37-65.

[38] Wolter, F. (1998). Fusions of modal logics revisited. In Advances in modal logic, Vol. 1 (Berlin, 1996) CSLI Lecture Notes (pp. 361-379).

[39] Wrónski, A. (1985). On a form of equational interpolation property. Foundations of Logic and Linguistics: Problems and their Solutions (G. Dorn and P. Weingartner, eds.), (pp. 23-29).

[40] Yorsh, G., \& Musuvathi, M. (2005). A combination method for generating interpolants. In Proc. of CADE-20 LNCS (pp. 353-368). 